\title{
Zwischen Traditionsbewahrung und Modernisierungsbereitschaft: Professorale Ansprüche an Lehre und Forschung
}

Die in Kap. 2 dargestellten medialen Diskurse vermitteln den Eindruck, dass man es innerhalb der Professorenschaft im Grunde mit zwei ungleich großen Gruppen - TraditionalistInnen und ModernisiererInnen - zu tun hat, die sich hinsichtlich ihres Selbstverständnisses und ihrer Deutungen universitärer Lehre und Forschung klar voneinander unterscheiden. Denn wir finden auf der einen Seite das journalistische Lob für diejenigen - bislang wenigen - ProfessorInnen, die den Reformgeist repräsentieren, und auf der anderen Seite Beiträge in der Zeitschrift Forschung und Lehre (F\&L), in denen mit einiger Vehemenz ,klassische', am Humboldtschen Bildungsideal und an Prinzipien der Grundlagenforschung orientierte Werte verteidigt werden.

In diesem Kapitel beginnen wir nun mit der Auswertung der Interviews, die wir mit Professorinnen und Professoren geführt haben. Zwar finden sich in diesem Datenmaterial auch reformkritische und auf Status-quo-Wahrung abzielende Äußerungen. Allein schon durch unsere Einbeziehung unterschiedlicher Disziplinen sowie jüngerer wie älterer ProfessorInnen zeigt sich insgesamt

\begin{abstract}
Alle in diesem und den folgenden Kapiteln wiedergegebenen Zitate wurden von uns zugunsten der besseren Lesbarkeit sprachlich behutsam geglättet. Inhaltliche Korrekturen an den Aussagen der InterviewpartnerInnen wurden hierbei nicht vorgenommen. Den Zitaten wird eine Interviewkennung zugeordnet, die aus der Angabe der Teildisziplin und der Fallnummer besteht. Dabei steht „LIT“ für die Neuere Deutsche Literatur, „BIO“ für die Zell- und Mikrobiologie, „JUR“ für das Strafrecht, „POL“ für die Vergleichende Regierungslehre und die Internationalen Beziehungen und „TECH“ für die Nachrichtenund Hochfrequenztechnik. Immer dann, wenn ein bestimmter Interviewpartner zitiert wird, wird beim ersten Zitat dessen Interviewkennung genannt. Alle weiteren folgenden Zitate ohne weitere Interviewkennung stammen dann ebenfalls von diesem Fall. Eine neue Interviewkennung wird erst angeführt, sobald eine andere interviewte Person zitiert wird.
\end{abstract}


jedoch ein differenziertes Bild. Dies wird zunächst anhand der Einschätzungen von „Bologna“, sodann generell anhand der subjektiven Ansprüche an Lehre und Forschung deutlich. Diese wurden in den Interviews nicht direkt erfragt, was auf ein gehöriges $\mathrm{Ma} ß$ an sozial erwünschtem Antwortverhalten hinausgelaufen wäre, sondern erschließen sich aus Beschreibungen positiver und negativer Lehrund Forschungserfahrungen, persönlicher Ziele und als wichtig angesehener Bedingungen für , gute' Forschung und Lehre.

\section{1 „Bologna“'-Reform: Keine einheitliche Gegnerschaft}

„Bologna“ ist nicht nur in der medialen Diskussion, sondern auch in unseren Interviews ein wichtiges Thema, wenn es um die Frage nach guten oder schlechten Rahmenbedingungen für die Realisierung eigener Ansprüche an die Lehre geht. Daher werden wir mit einem kurzen Überblick über professorale Deutungen der unter diesem Begriff firmierenden Studienreform beginnen. In unseren Interviews findet sich zwar auch eine eher allgemeine „Bologna“Kritik. Wir gründen unsere Darstellung jedoch vor allem auf Äußerungen, mit denen ProfessorInnen die Veränderungen - etwa die Umstellung auf Bachelor und Master, die Modularisierung der Studiengänge oder neue Bewertungs- und Prüfungsformate - konkreter auf ihr eigenes berufliches Handeln und eigene Ansprüche an die Lehre beziehen.

\section{Zeitliche Mehrbelastung und Entwertung professoraler Kompetenzen}

Obwohl sich in unseren Daten keineswegs ein einhelliges Votum gegen die „Bologna“-Reformen widerspiegelt, werden von ProfessorInnen verschiedene Veränderungen kritisch betrachtet. Dazu gehört der oft genannte Punkt einer zeitlichen Vereinnahmung durch neue bürokratische Anforderungen und Abstimmungserfordernisse. So spricht ein Literaturwissenschaftler die Vielzahl neuer Studiengänge an, durch die sich seine „Arbeitsbedingungen deutlich“ „und zwar nicht zum Besseren“ - gewandelt hätten:

„Man kann das zum Teil gar nicht mehr bedienen und die Termine einhalten. Jeder Studiengang hat eigene Anmeldetermine, Abgabefristen, die in unser zentrales System eingetragen werden müssen. Das kostet furchtbar viel Zeit und eigentlich, wenn man es genau nimmt, dürfte man sich aus dem Büro überhaupt nicht mehr wegbewegen, weil garantiert irgendein Termin ansteht, der bedient werden muss.“ (LIT8) 
Hinzu kommt, dass „Bologna“ von nicht wenigen ProfessorInnen mit anhaltenden, sozusagen nicht enden wollenden Veränderungen verbunden wird. Eine Literaturwissenschaftlerin klagt über einen ,unentwegte[n] Reformprozess“, durch den sie und ihre KollegInnen ,,permanent dabei“ seien, ,extra zu verwalten" (LIT13). Auch ein Politikwissenschaftler geht von einer Situation aus, die weiterhin von ,viel Kopfzerbrechen“ (POL13) geprägt sei. Aus seiner Sicht befänden er und seine KollegInnen sich

„immer noch in der Phase, wo man die Effekte der Umstellung von den alten Studiengängen auf die neuen merkt, weil man nicht genau weiß: Was gehört in den Bachelor, was gehört in den Master? Und dann reformiert man und muss sehr schnell irgendwie nachjustieren, weil man merkt, man muss sich irgendwie klar werden, welche Inhalte gehören in den Bachelor, welche in den Master. Die Prüfungsanforderungen, das Denken in Modulen, das führt zu viel Kopfzerbrechen. [...] Man muss sich besser koordinieren und abstimmen.“

Solche Beschreibungen müssen keineswegs Ausdruck einer grundsätzlich kritischen Sicht auf die Studienreform sein. Sie zeigen aber, dass ProfessorInnen eine Zunahme organisationaler Anforderungen registrieren. ${ }^{1}$

Teilweise werden die vermehrten formalen Vorgaben jedoch auch als Entwertung einer inhaltlich-fachlichen Auseinandersetzung gedeutet. So spricht etwa ein Nachrichtentechniker mit Blick auf die Akkreditierungsverfahren von „sinnloser Bürokratie“, die „,nichts an Qualität“ bringe. Seine KollegInnen und er formulierten die geforderten Berichte so, dass „es schön kling[e]“, also der gewünschten Semantik entspreche. Letztlich werde durch dieses Verfahren aber die professorale Verantwortung für die Lehre beschnitten:

\begin{abstract}
„Also man soll doch den Professoren ein bisschen mehr zutrauen, dass die wissen, wie man ein Studium organisiert. [...] Auch wenn es vielleicht nicht alle machen. Das ist ja immer der Vorwurf: Professoren kümmern sich um ihre Nebentätigkeiten, um ihre Forschung, aber nicht um die Lehre; aber es gibt genügend Engagierte in der Lehre, die dann auch diese Aufgaben übernehmen und diesen Studiengang besser machen, als wenn man da so einen Rahmen von außen vorgibt. Und die Akkreditierung: Da werden Formalitäten überprüft letztendlich, über Inhalte ging es da fast gar nicht." (TECH3)
\end{abstract}

\footnotetext{
${ }^{1} \mathrm{Zu}$ den hohen Transaktionskosten der Hochschulreformen siehe auch Jörg Bogumil et al. (2015) sowie Peer Pasternack et al. (2017, S. 5, 75-79) die in ihren Befragungen entsprechende Einschätzungen betroffener Akteure ermittelt haben.
} 
Der Nachrichtentechniker äußert somit mehr als eine Bürokratiekritik. Er nimmt eine Verkehrung der Rollen wahr, im Zuge derer sich die in Bezug auf die Lehrinhalte eigentlich kompetenten ProfessorInnen an einen „von außen“ vorgegebenen „Rahmen“ anpassen müssten. Er diagnostiziert hier etwas, das von der Arbeitssoziologie unter dem Begriff der „Entsubjektivierung“ lange Zeit in ganz anderen Berufsfeldern untersucht wurde, wo Tätigkeiten wie Fließbandarbeit immer größeren Standardisierungen unterworfen wurden (Kern und Schumann 1970, 1984; Braverman 1977; Bolte und Treutner 1983). ${ }^{2}$

Neben den Akkreditierungsverfahren wird auch die Einflussnahme der eigenen Organisation als eine Verschiebung der Kompetenzen gedeutet. Dies trifft etwa auf einen Literaturwissenschaftler zu, der die Zunahme von organisationalen Vorgaben als „Gängelband“ für die ProfessorInnen bewertet:

\begin{abstract}
„Wenn man sich anguckt, wie das jetzt gemacht wird. Fangen wir mal beim Detail an! Dass Studierende, was ja auch sinnvoll ist, zum ganz bestimmten Zeitpunkt ihre Note haben müssen, und dass ich dann daraus zurückrechne, wann Themen gestellt werden müssen, wann und wie schnell Arbeiten korrigiert werden müssen: Dann kann man schon an diesem Detail feststellen, dass es einen massiven Prozess gibt, die Verantwortlichkeit des Amtes eines Professors oder einer Professorin wirklich zu unterwandern. Also Professorinnen und Professoren sind nicht mehr selbst verantwortlich für ihr Tun, sondern sind am Gängelband organisationeller Verpflichtungen, und das ist in der Tat wirklich massiv." (LIT9)
\end{abstract}

Obgleich der Literaturwissenschaftler eine Verbindlichkeit für die Studierenden für „sinnvoll“ hält, überwiegt seine Kritik an einer auf stärkere Formalisierung abzielenden Governance. Aus seiner Sicht wird dadurch das Amtsethos von ProfessorInnen, das auf einer freien, eigenverantwortlichen Aufgabenerfüllung beruht, ,unterwandert“.

\title{
„Bologna“ als Bedrohung der inhaltlichen Qualität universitärer Lehre?
}

Wenn man sich die professoralen Beiträge in F\&L vergegenwärtigt, ging es bei der Auseinandersetzung mit „Bologna“ vor allem auch um ein befürchtetes oder

\footnotetext{
${ }^{2}$ In ihrer Untersuchung von Ökonomisierungsprozessen in der Bildungsarbeit gelangen Karin Lohr et al. (2013, S. 239-244) zu dem Fazit, dass die verstärkt eingesetzten standardisierten Verfahren in der Lehre Beschränkungen der fachlich-professionellen Handlungsfreiheit und damit Momente der „Entsubjektivierung“ erzeugen. Aufgrund der weiterhin großen Handlungsspielräume der BildungsarbeiterInnen und ihrer Mitgestaltung an diesen Verfahren werden diese Momente jedoch als bislang noch schwach eingestuft.
} 
schon diagnostiziertes Ende spezifischer inhaltlicher Qualitäten universitärer Lehre. Schließlich wurde die Studienreform dort unter anderem mit einer Entwicklung zur „Massenfertigung“ (Loer, F\&L 04/2012, S. 289) und einer zunehmenden Entfernung von klassischen Bildungsidealen gleichgesetzt. In unseren Interviews wird jedoch nur selten eine so weitgehende Krisendiagnose geäußert, und es finden sich auch positivere oder zumindest gelassene Deutungen der Veränderungen.

Als besonders negativ werden die Folgen für die Lehre von einem älteren Professor für Neuere Deutsche Literatur beschrieben. Dieser geht von dem kontrastiven Vergleich mit einer Vergangenheit aus, in der das „gemeinsame Lernen und Forschen“ noch von „Freiheit" bestimmt gewesen sei (LIT1). So habe er bei einem Professor studiert, der ,enthusiastisch für sein Fach hinreißende Dinge mit Studenten machte" und eine Art Vorbild für ihn wurde. Durch die „Bologna“-Reformen müsse er sich als Lehrender nun aber erst gegenüber zu strikten Vorgaben - „,sehr eng getakteten“ Stundenplänen der Studierenden und der „unerschütterlich fixierte[n] Dauer einer Seminarsitzung“ - mühsam seine „Freiräume erkämpfen“. Auch hier klingt, wie zuvor beim Nachrichtentechniker, „Entsubjektivierung“ an. Die Bereitschaft, sich von einem inhaltlichen Interesse leiten zu lassen, fordere beiden Seiten - ihm selbst wie auch den Studierenden unangemessene Zugeständnisse $a b$ :

„Wir machen es dann als Privatveranstaltungen. Es ist auch ganz traurig, dass ich eine Veranstaltung wie dieses Lyrikseminar außerhalb meiner Arbeitszeit mache und dass keiner der beteiligten Studierenden dafür einen Punkt bekommt, obwohl es vielleicht eines der lehrreichsten Seminare ihrer Ausbildungszeit gewesen ist.“

Folgerichtig könne die Lösung nur in einer stärkeren Flexibilität der Lehre - einer größeren Lehr- und Lernfreiheit - bestehen:

„Ich würde gerne einen Studienplan sehen, in dem es viel weniger fest vorgeschriebene Anforderungen gibt, diese dann aber flexibler gelernt werden können. Sodass wir sagen können, wie das in meiner Studienzeit bei meinen Lehrern noch möglich war: ,Wir machen dieses Seminar hier, weil der Text so komplex ist oder weil so viele Leute da sind oder weil das Ergebnis so vielversprechend ist. Wir machen jetzt mal drei Stunden statt zwei oder vier. Uns fällt jetzt ein: Wir machen am Wochenende einen Ausflug ins Goethehaus und schauen uns die Handschriften dort an. ' Das wäre sehr förderlich für das Erreichen der Ausbildungsziele, wenn wir so was machen könnten. Es ist vollkommen utopisch, so etwas zu machen.“

Der Literaturwissenschaftler geht von einem deutlichen Widerspruch zwischen „Bologna“ und dem Erreichen eigentlich wichtiger Lehrziele aus. Die „Schere 
zwischen dem“, worauf es in der „Ausbildung junger Leute zu sehr guten Literaturwissenschaftlerinnen und -wissenschaftlern" wirklich ankomme, und den Regelungen der neuen Studiengänge „öffne[] sich“, wie er an anderer Stelle formuliert, „weiter, und zwar dramatisch weiter“".

In der Folge zeichnen sich negative Konsequenzen für die Lehrmotivation ab. So unterscheidet eine Reihe von ProfessorInnen im Zuge von „Bologna“ zunehmend zwischen einer wenig sinnstiftenden Pflichtlehre und seltener werdenden Veranstaltungen, in denen sich forschungsorientierter Austausch realisieren lässt:

\begin{abstract}
„Das sind in der Tat die interessanten Seminare. Was wir jetzt machen, wir haben jetzt wieder ein Oberseminar, das heißt einfach so oder muss auch, glaube ich, so heißen, damit wir einen Raum kriegen, was auch irgendwie eigenartig ist. Aber das ist völlig frei von Studiengangszwängen, wo wir uns einfach irgendetwas vornehmen, um zu sagen: ,Ach, das finden wir interessant, das diskutieren wir mal, gucken wir mal, ob daraus irgendwie eine Projektidee entsteht. ““(LIT9)
\end{abstract}

Mit der Unterscheidung zwischen „interessanten Seminaren“ und weniger sinnstiftenden Veranstaltungen geht teilweise einher, eigenen Qualitätsmaßstäben in Bezug auf die Lehre nicht mehr durchgängig folgen zu können. So äußert derselbe Literaturwissenschaftler, "die Lust“" an einer zeitaufwendigen Vorbereitung zu verlieren, da er mit seinen inhaltlichen Ansprüchen immer seltener auf ein entsprechendes „Publikum“ stoße. Durch „Bologna“ und „das Damoklesschwert" der ECTS-Punkte seien viele Studierende mehr als früher an einer vorstrukturierten Wissensvermittlung interessiert. Ihre Bereitschaft, sich auf komplexere Lern- und Aneignungsprozesse einzulassen, sinke entsprechend:

„Es ist natürlich klar: Warum soll man so viel Zeit opfern, um schwierige Texte zu lesen, um dann eine Note zu bekommen, die, das kann man dann prozentual ausrechnen, irgendwie so im einstelligen Bereich liegt bezogen auf das Gesamtstudium."

Solche Veränderungen der studentischen „Mentalitäten“3 ${ }^{\text {3 }}$ werden nicht nur von GeisteswissenschaftlerInnen thematisiert. So konstatiert eine ältere Professorin

\footnotetext{
${ }^{3}$ Siehe hierzu auch die Ergebnisse von Harald Schomburg et al. (2012, S. 90-92): Die Mehrheit der Lehrenden an Universitäten stimmte der Aussage zu, dass die intrinsische Motivation der Studierenden im Zuge von „Bologna“ abgenommen habe.
} 
der Zellbiologie, dass sich durch die stärkere Verschulung der Bachelor-Studiengänge insbesondere die Haltung von StudienanfängerInnen gewandelt habe:

\begin{abstract}
„Das war wirklich ein großer Umbruch. Und ich meine, ich bin ja auch schon etwas älter, ich stehe dem durchaus sehr kritisch gegenüber. Weil einfach, sagen wir mal, die Selbstverantwortlichkeit der Studierenden für ihre Ausbildung darunter sehr, sehr stark gelitten hat. [...] Also zumindest der Bachelor-Studiengang ist sehr stark verschult. [...] Man kann nicht mehr davon ausgehen, dass die Leute sich für das interessieren, was man da macht, sondern die meisten natürlich nicht. Die müssen es nur machen, damit sie ihre Punkte kriegen und ihre Klausuren bestehen." (BIO3)
\end{abstract}

Ihren Vergleichsmaßstab bildet die größere Wahlfreiheit in den Diplomstudiengängen, die es den Studierenden schon früh ermöglicht habe, sich „selbstverantwortlich" an den eigenen inhaltlichen Interessen zu orientieren. Auch ein erfahrener Fachkollege einer anderen Universität spricht davon, dass Studierende die Veranstaltungen vermehrt „nur noch als Pflicht“ wahrnähmen und ihr „Interesse am Stoff rasant gesunken“ sei (BIO2).

Neben motivationalen Problemen sehen ZellbiologInnen häufiger auch einen Qualitätsverlust des vermittelten Fachwissens. Durch die modularisierten Prüfungen würden die jeweiligen Inhalte zunehmend ,abgehakt“ (BIO3) oder „als Pakete behandelt“ (BIO1), die nacheinander ,zu schnüren“ seien. Hierdurch werde ein Grundverständnis der Zusammenhänge verhindert, so dass „einfach kein Gesamtkonzept Biologie [...] mehr im Kopf“ entstehe, wie es „die Diplomleute" noch gehabt hätten (BIO3).

Wie erwähnt, finden wir in den Interviews jedoch ebenfalls positive oder zumindest gelassene Einschätzungen der Folgen von „Bologna“ für die Lehre. Eine besonders klare Gegenposition zu den KritikerInnen nimmt etwa ein Nachrichtentechniker ein, der die Studienreform vor allem mit Verbesserungschancen verbindet. Er wertet es als positive Entwicklung, dass mit der Aufteilung in Bachelor und Master eine „Sollbruchstelle“ entstanden sei, durch die Studierende nach nur wenigen Jahren eine „Ausbildung nachweisen“ und in das Berufsleben starten könnten:

„Und ich denke, das ist gut so. Vorher hat man das Vordiplom gehabt, das war eigentlich nichts wert. Also hier in der Elektrotechnik war das nichts wert. Man musste entweder das gesamte Studium machen oder man ist gescheitert." (TECH2)

Dass er im Gegenzug seine Bachelor-Veranstaltungen stärker an den Erfordernissen eines potenziell früheren Arbeitsmarkteintrittes $\mathrm{zu}$ orientieren habe, bewertet er als sinnvoll: 
,[J]etzt muss ich vielleicht meine Vorlesung umstellen, damit ich diesen BachelorStudiengang so bediene, wie das die Leute brauchen. Sie brauchen eben nicht die tiefen theoretischen Kenntnisse, sondern sie müssen von allem mal was gehört haben, damit sie nachher in die Industrie gehen. Weil, die Industrie wird sie so oder so anders formen. Das ist so.“

Anstatt von einem besseren früheren Status quo auszugehen, folgt der Nachrichtentechniker einer Lesart, die auch den wissenschafts- und reformpolitischen Diskurs bestimmt. Die universitäre Lehre muss demgemäß stärker in Relation zu den Anforderungen der Arbeitswelt gesehen werden.

Mit seinen eindeutig positiven und am Ziel der „Employability“ orientierten Deutungen gehört der Nachrichtentechniker innerhalb des Samples eher zu den Ausnahmen. Es finden sich jedoch selbst bei den - tendenziell „Bologna“kritischeren - GermanistikprofessorInnen Beispiele für eine zumindest abwägende Perspektive auf die Studienreform. Erstens würden mit dieser Lehrende nun stärker ,als didaktisch Handelnde in den Blick genommen“ (LIT1), wie ein ansonsten die Studienreform kritisierender Literaturwissenschaftler positiv vermerkt. Zweitens wird mit der stärkeren Strukturierung der Lehrinhalte auch ein Qualitätsgewinn verbunden. Eine Fachkollegin erläutert:

\begin{abstract}
„Es hat in unserem Fach, also Neuere Deutsche Literatur, auch gebracht, dass wir uns selber, also die Lehrenden, viel klarer darüber werden mussten, was sind eigentlich unsere Standards? Was muss eigentlich ein Student, eine Studentin wissen, wenn sie drei Jahre oder vier Jahre Germanistik studiert haben? [...] Das war vorher sehr freihändig, da hat das jeder irgendwie gemacht, und da musste man sich jetzt einigen, und das finde ich eine sehr gute Lösung, dass wir sozusagen die Einführung, die erste Stufe des Studiums, reglementiert haben. Also reglementiert haben in dem Sinne, dass wir sagen, alle müssen das Gleiche machen, und dann kann sich jeder nachher so entwickeln und aussuchen, was er oder sie will. Das wäre eine Verschulung in einem Sinne, den ich durchaus positiv finde - nämlich: Wir müssen uns Rechenschaft darüber abgeben, was sollen die wissen? Welche Kompetenzen sollen sie entfalten? Im Schulalltag ist das ganz normal, an den Unis war es oft nicht der Fall.“ (LIT5)
\end{abstract}

Anstatt von einer Dichotomie zwischen positiv bestimmter Lehr- und Lernfreiheit und kritikwürdiger Verschulung auszugehen, betrachtet die Germanistin die Veränderungen in differenzierter Weise. Sofern sich die Reglementierung auf „die erste Stufe des Studiums“ beschränke, könne „Bologna“ dem Ziel dienen, über einheitliche „Standards“ eine bessere Basis für die anschließend größere Lernfreiheit der Studierenden zu vermitteln. In ähnlicher Weise argumentiert auch eine jüngere Fachkollegin, die die Vorteile einer stärkeren Strukturierung 
noch konkreter auf ihr eigenes berufliches Handeln bezieht. So gebe ihr die Modularisierung mehr Orientierung, von welchen Kompetenzen der Studierenden sie in ihren Lehrveranstaltungen ausgehen könne - ,auf welcher Stufe“ (LIT2) sie sich also jeweils befinde.

In unserem Überblick spiegelt sich somit zum einen wider, dass „Bologna“ durchaus als noch nicht abgeschlossene Reform wahrgenommen wird. Denn anders als man aufgrund des Zeitpunkts unserer Interviews hätte vermuten können, ist häufiger noch die Rede von Prozessen der Anpassung oder Nachjustierung, die den beruflichen Alltag begleiten. Zum anderen deutet sich in den Äußerungen zu den Reformen bereits ein Spektrum unterschiedlicher Ansprüche an die Lehre an. Dies reicht - zugespitzt - vom klassischen Ideal einer an Humboldt orientierten Lehr- und Lernfreiheit bis zu einer Vorbereitung der Studierenden auf den Arbeitsmarkt. Entsprechend wurde „Bologna“ mit eher förderlichen oder aber hinderlichen Bedingungen für die Realisierung eigener Ansprüche an die Lehre in Verbindung gebracht.

Im folgenden Abschnitt werden wir die professoralen Ansprüche an die Lehre ausführlicher und mit Blick auf disziplinäre Unterschiede darstellen. Die Gefährdung dieser Ansprüche wird in den Interviews weniger an Leistungsbewertungen in der Lehre festgemacht. Wie wir sehen werden, gerät die Lehre eher durch solche neuen Leistungsbewertungen und -kriterien unter Druck, die an die Forschung angelegt werden.

\section{2 ,Gute' Lehre}

Zunächst gehen wir von dem Anspruch aus, Lehre als einen interaktiven Prozess zu gestalten, der sich deutlich von einer unidirektionalen Wissensvermittlung qua ,Unterricht“ unterscheidet. In den darauf folgenden Abschnitten werden wir dann näher betrachten, inwieweit sich ProfessorInnen weiterhin an dem für die universitäre Lehre klassischen Anspruch einer Integration von Forschung und Lehre orientieren und wie sie ihre Lehrziele zwischen Wissenschaft, Arbeitsmarkt und erweiterten Bildungsidealen verorten.

\section{Interaktive Lehr- und Lernprozesse}

Der Anspruch, Lehre als interaktiven, möglichst dialogischen Prozess zu gestalten, wird in besonderer Weise von jenem älteren Literaturwissenschaftler herausgestellt, der die „Bologna“-Reformen - wie in Kap. 3.1 dargestellt - als massiven Einschnitt in die Lehr- und Lernfreiheit deutet. Positive Beispiele für 
Lehrveranstaltungen sind für ihn vor allem Seminare, in denen eine anspruchsvolle gemeinsame Arbeit an literarischen Texten möglich ist. Hierzu gehörte etwa ein Hauptseminar, in dessen Vorfeld die Studierenden einen umfangreichen Roman lesen mussten und in dem sich dann durch die von allen geteilte „Souveränität einer wirklich guten Textkenntnis“ eine Diskussion „über die interessantesten und schwierigsten Aspekte" habe entwickeln können:

\begin{abstract}
„Mein Vorsprung bestand nur noch darin, dass ich mich mehr in der [Forschungsthema]-Forschung und in der Textanalyse auskannte. Aber im Text dieses Werkes waren wir eigentlich alle gemeinsam da. Ich glaube, das hat allen sehr gut gefallen, die in diesem Seminar waren, weil man auch nach diesem Seminar nicht mehr gefragt hätte, zu welchem Ende studiert man Literaturwissenschaften? Eben, um solche wunderbaren Werke zu verstehen und durch sie die Welt besser zu verstehen." (LIT1)
\end{abstract}

Der Literaturwissenschaftler hebt hervor, dass die Hierarchie zwischen lehrendem Professor und lernenden Studierenden in den Hintergrund treten konnte. Trotz seines größeren Fachwissens nahm er keine anleitende oder motivierende Rolle ein, da er auf TeilnehmerInnen traf, die sich auf ein komplexes Werk und einen davon ausgehenden Bildungsprozess einließen. So ergab sich eine Gemeinschaft des Lehrenden und der Lernenden, in der sich eine Einheit von Forschung und Lehre einstellte - ganz im Sinne Wilhelm von Humboldts (1809-10, S. 230):

\begin{abstract}
„Es ist [...] eine Eigenthümlichkeit der höheren wissenschaftlichen Anstalten, dass sie die Wissenschaft immer als ein noch nicht ganz aufgelöstes Problem behandeln und daher immer im Forschen bleiben, da die Schule es nur mit fertigen und abgemachten Kenntnissen zu thun hat und lernt. Das Verhältniss zwischen Lehrer und Schüler wird daher durchaus ein anderes als vorher. Der erstere ist nicht für die letzteren, Beide sind für die Wissenschaft da [...].“
\end{abstract}

An anderer Stelle betont der Literaturwissenschaftler zwar, dass die Vermittlung von Grundlagenwissen ebenfalls „nicht geringgeschätzt werden“ sollte. Lehrveranstaltungen, die er selbst als „,schön, befriedigend und sinnvoll“ erlebt, sind jedoch deutlich voraussetzungsvoller.

Selbst wenn man zunächst noch bei den Literaturwissenschaften bleibt, zeigen sich aber auch hier bereits Unterschiede. Dass sich die Studierenden aktiv beteiligen und auf die jeweiligen Gegenstände einlassen, wird zwar durchgängig als wichtig erachtet. Wie sich anhand des Beispiels einer jüngeren Professorin illustrieren lässt, kann sich dieser Anspruch aber ebenso mit dem Selbstverständ- 
nis einer stärker anleitenden Lehrenden verbinden. So äußert die Professorin, dass es ihr ,immer wichtig“ sei, ,auch Erstsemester und Zweitsemester zu unterrichten“, um sehen zu können, mit welchen Lektüreerfahrungen und welchem „Medienverhalten“ StudienanfängerInnen in ihre ersten Veranstaltungen kämen. Ihre Aufgabe sieht sie nicht zuletzt darin, sich an den Potenzialen, aber auch an den bestehenden Grenzen der Studierenden zu orientieren:

\begin{abstract}
„Das heißt einerseits, stark leitend zu sein. Ich glaube schon, dass es wichtig ist, anleitend zu sein. Und Standards und Kriterien anzugeben, mit denen sinnvollerweise beispielsweise Texte [...] gedeutet werden können. Und andererseits aber eben auch dem Anderen den Freiraum zu lassen, bestimmte Dinge überhaupt erst zu entwickeln. Das ist teilweise gar nicht so einfach, und deswegen bin ich immer so gerne im ersten Semester, um noch immer zu bedenken, wo sind eigentlich die Schwierigkeiten? Warum sind bestimmte Fragestellungen für Leute, die am Anfang sind, noch keine Routine? Warum haben sie Schwierigkeiten, Sekundärtexte zu lesen?"(LIT2)
\end{abstract}

Die Literaturwissenschaftlerin geht von einer Balance zwischen „Freiraum“ und Anleitung aus, um zugleich zwei Zielen gerecht werden zu können: der Vermittlung fachlicher „Standards“ und der Begleitung ihrer Studierenden bei der allmählichen Entwicklung einer für sie neuen Perspektive. Dass sie dabei noch auf spürbare „Schwierigkeiten“ trifft, ist für sie weder demotivierend noch enttäuschend - eher im Gegenteil: Die Literaturwissenschaftlerin sieht es gerade als positive Herausforderung, ,wechselseitig im Gespräch“ mit den Studierenden eine allmähliche „Verwissenschaftlichung des Blicks“ einzuüben. Dieser Prozess sei ,ganz, ganz bemerkenswert" und interessant. Insofern handelt es sich bei der Arbeit mit Bachelor-Studierenden für sie keineswegs um eine ungeliebte Pflicht.

Vergleichbare Deutungen finden sich ebenfalls bei einer jüngeren Politikwissenschaftlerin. Auch diese betont ihren Anspruch, Studierende bei der Aneignung einer für sie neuen Form der Reflexion zu unterstützen. Wichtig sei hierbei aber noch, die Teilnehmenden zu einer aktiven Mitarbeit zu verpflichten:

,[I]ch [verlange] in der Regel von ihnen, dass sie sich halt beteiligen, dass sie auf jeden Fall die Texte gelesen haben und dass sie einzelne Leistungen erbringen müssen, die ich auch kontrolliere. Das funktioniert bisher erstaunlich gut. Das machen die auch."(POL7)

Eine solche Leistungskontrolle könnte ebenso als Ausdruck einer negativen Verschulung der universitären Lehre gedeutet werden. Die Politikwissenschaftlerin sieht darin jedoch die Basis für eine verbindliche Zusammenarbeit. So gebe sie 
im Gegenzug „,immer Feedback auf jede Teilaufgabe“, damit die Studierenden einschätzen könnten, an welchem „Standard“ sie sich orientieren sollten.

Die Professorin geht daneben durchaus auf Widerstände ein, die auch in anderen Interviews genannt werden. So müsse man sich gerade vor StudienanfängerInnen „rechtfertigen“, wenn man keinen unidirektionalen „Unterricht“ biete und auf mehr als rein „klausurrelevantes“ Wissen Wert lege. Zugleich ist die Lehre im Bachelor für sie aber „schon auch interessanter“, da die Studierenden noch näher an ihrer ,ursprünglichen Motivation“ und weniger „abgeklärt" seien. Besonders erfreuten sie daher auch die „Aha-Moment(e)“ wenn die Studierenden also verstünden, ,was die Wissenschaft da dran ist“".

Als besonders positiv werden folglich solche Lehrveranstaltungen bzw. -situationen beschrieben, die durch wechselseitigen Austausch und aktive Beteiligung der Studierenden gekennzeichnet sind. Zugleich zeigt sich, dass sich jüngere ProfessorInnen seltener an dem Ideal einer gemeinsamen Reflexion auf Augenhöhe orientieren und ihre Rolle als Lehrende auch über eine didaktische Anleitung von Lernprozessen und die Vermittlung bestimmter "Standards“ definieren. Interessant ist nun die Frage, welche Rolle der Anspruch interaktiver Lehr- und Lernprozesse für solche ProfessorInnen spielt, in deren Disziplinen Vorlesungen einen größeren Raum als in den Geistes- und Sozialwissenschaften einnehmen oder die Lehre zusätzlich, wie in den Rechtswissenschaften, per se durch eine stärker standardisierte, examensvorbereitende Wissensvermittlung geprägt ist.

Wichtig ist zunächst, dass der Anspruch, über die erfolgreiche Vermittlung von Lehrinhalten hinaus auch dialogische Anteile zu integrieren, grundsätzlich geteilt wird. Dies zeigt sich etwa bei einem Professor für Hochfrequenztechnik, der kleinere Vorlesungen aus diesem Grund als besonders positiv erlebt:

„Die sind mit Leib und Seele dabei. Ich überziehe auch fast immer. Das stört keinen. Die gehen dann nicht, sondern die machen auch eine halbe Stunde länger, wenn es sie interessiert. Und diese Zusammenarbeit ist eben das, was ich faszinierend finde. Deshalb macht mir Lehre auch eben wirklich Spaß. Weil es halt Spaß macht, mit den Leuten zu diskutieren, zu sehen, was können die schon, wo haben die Lücken? Was kann ich denen noch mit auf den Weg geben?" (TECH1)

Große Vorlesungen, in denen er vor einer ,anonyme[n] graue[n] Masse“ stehe und nur „selten mal Fragen“ von den Teilnehmenden kämen, deutet er vor allem pflichtethisch. Unidirektionale Wissensvermittlung müsse auch ,gemacht werden“, stelle „eben halt die Schattenseite der Medaille“ dar. „Wirkliche Lehre“ aber fange erst an, wenn ein Dialog mit den Studierenden möglich sei. 
Bei den StrafrechtlerInnen finden wir eine solche Unterscheidung zwischen „wirklicher“ Lehre und notwendigen Pflichtveranstaltungen seltener. Dies ist vor allem der bereits erwähnten disziplinären Besonderheit - eine stärker vorstrukturierte und am Staatsexamen orientierte Wissensvermittlung - geschuldet. Ein geringerer diskursiver Anteil stellt für die ProfessorInnen insofern eher den Normalfall als die „Schattenseite der Medaille“ dar. So sieht eine jüngere Strafrechtlerin die wesentliche Herausforderung ihrer Lehre darin, tendenziell ,zu viel Stoff in zu kurzer Zeit“ vermitteln zu müssen, da im Examen oftmals „kleinliche[] Details“ geprüft würden (JUR3). Die Studierenden stünden im Gegenzug unter einem großen „Druck“ und fokussierten vor allem „das eine Ziel: irgendwie durchkommen“. Ein ebenfalls noch jüngerer Fachkollege äußert einen ähnlichen Eindruck. Die „Allermeisten“ seien „stark orientiert auf das Examen“, sodass

„im Grunde all das, was man ihnen dann in den Vorlesungen, in Seminaren bietet, durchaus auch darauf abgescannt wird, was davon kann ich später mal gebrauchen, was ist jetzt prüfungsrelevant, was ist examensrelevant. [...] Was freilich vor der Menge des Stoffs und vor dieser überragenden Bedeutung der Examensnote aus meiner Sicht verständlich ist.“ (JUR12)

Er verbindet damit also keine Kritik an den Studierenden. Während einige der älteren FachkollegInnen die heutigen Studierenden im Vergleich durchaus als eindimensionaler wahrnehmen, ist die beschriebene Haltung für den zitierten Strafrechtler angesichts der Struktur eines rechtswissenschaftlichen Studiums „verständlich“ und habe sich über die Jahre nicht wesentlich verändert. „Diese Examensorientierung" und das damit „ein bisschen verbundene Scheuklappendenken“ seien bereits zu seiner eigenen Studienzeit ,,vor 20 Jahren nicht großartig anders" gewesen. ${ }^{4}$

Insgesamt überwiegen in den Rechtswissenschaften Deutungen, nach denen die Aufgabe der ProfessorInnen vor allem darin bestehe, Inhalte in eine lernbare Form zu bringen. Auch wenn die befragten JuristInnen das „Scheuklappendenken“ der Studierenden kritisieren, akzeptieren sie diesbezügliche Einschränkungen ihrer Lehrfreiheit. So beschreibt die eben zitierte Strafrechtlerin ihre Lehre nicht zuletzt darüber, „den Stoff zu strukturieren“ und die „Komplexität“ mancher Fragen ,in einfache Schritte aufzusplitten“ (JUR3). Sie sei mit ihren Vorlesungen „desto zufriedener, je besser sie Gedanken aufbauen“

\footnotetext{
${ }^{4}$ Zur eingeschränkten Lehr- und Lernfreiheit in professionsorientierten Studiengängen siehe Götz Fabry und Christian Schirlo (2016) am Beispiel der Humanmedizin.
} 
könne. Dennoch findet sich bei ihr zugleich der Anspruch, in ein wechselseitiges Verhältnis mit den Studierenden zu treten. So betont die Strafrechtlerin auch, dass ihr „sprachliches Können“ immer „stark vom Gegenüber“ abhänge. Es „mach[e]“ sie „,besser“, wenn sie „im Raum“ spüre, dass „die Leute drinnen“ seien - wenn sie bei den Studierenden also eine, wie es Uta Liebeskind (2011, S. 205) bezeichnet, ,tatsächlich anteilnehmende Teilnahme" wahrnimmt. Zudem versucht die Strafrechtlerin ,immer wieder“, die unidirektionale Struktur ihrer Vorlesungen bewusst durch konkrete Fragen zu durchbrechen und zumindest „einzelne Personen“ dialogisch „einzubeziehen“ (JUR3). Während in den großen Vorlesungen weiterhin ,die schweigende Masse“ dominiere, sei die aktive Beteiligung bei einer geringeren Studierendenzahl eher möglich:

\begin{abstract}
„Auch von mir aus kommt da viel mehr kommunikatives Anliegen. Da habe ich zum Beispiel begonnen mit so einem kleinen Multiple-Choice-Test, der das, was wir letzte Woche hatten, wiederholt. Ich habe dazu gesagt: ,So, jetzt sprechen wir den Multiple-Choice-Test durch." Jeder hat dann irgendwie was gesagt. Jeder hat sich damit angesprochen gefühlt."
\end{abstract}

Mit der Durchführung und Nachbesprechung von Multiple-Choice-Tests nennt die Strafrechtlerin zwar ein didaktisches Instrument, das vergleichsweise wenig Raum für Diskussionen lässt. Gleichwohl wird deutlich, dass sie es als Mehrwert wahrnimmt, ihre Lehre auf diesem Wege „kommunikativer“ gestalten zu können.

Der ebenfalls schon zitierte Fachkollege geht mit seinem Anspruch etwas weiter, indem er in seinen Vorlesungen auch Diskussionen anzuregen versucht. Um diesen Prozess zu fördern, stelle er den Studierenden ,ausführliche Lehrskripte“ zur Verfügung - so seien die TeilnehmerInnen eher „frei“ und in der Lage, „sich das anzuschauen, anzuhören und aber eben dann auch mitdiskutieren zu können“"(JUR12). Der Strafrechtler folgt mit den Lehrskripten insofern weniger einer Dienstleistungsnorm oder -erwartung als seinem eigenen Anspruch, die Studierenden aktiv zu beteiligen. „Am meisten Spaß“ machten ihm letztlich die wenigen Lektürekurse, in denen der Raum für eine offene Diskussion vorhanden sei:

\footnotetext{
„Da nimmt man sich die Texte, auf die man Lust hat, und dann schaut man mal, wie weit man kommt, und beißt sich dann fest oder geht dann eben auch schnell und zügig weiter. Da hat man im Grunde noch so diese alte Diskussionskultur, die man so in diesen Seminaren selbst in den 70er, 80er Jahren erlebt hat [...].“
}

Die Zufriedenheit mit der Lehre wird also in starkem Maße von der Beteiligung und Haltung der Studierenden abhängig gemacht. Grenzen für die Umsetzung 
interaktiver Anteile werden zwar thematisiert. Es bleiben aber nicht zuletzt jene Lehrveranstaltungen in positiver Erinnerung, in denen mehr möglich war. Die Aktivität der Studierenden wird von ProfessorInnen insofern als ,konstitutiver Part universitärer Lehre“ (Liebeskind 2011, S. 222) gesehen.

\section{Integration von Forschung und Lehre}

Ein wichtiger Anspruch, der sich in den meisten Interviews findet, ist eine Integration von Forschung und Lehre. Dieser Anspruch deutete sich bereits im vorangegangenen Abschnitt an, wenn etwa die ProfessorInnen aus den Literaturund Politikwissenschaften positive Lehrerfahrungen an der erfolgreichen Vermittlung einer wissenschaftlichen Perspektive festmachten.

Hinsichtlich der Möglichkeiten und Formen einer Integration von Forschung und Lehre zeigen sich disziplinäre Unterschiede, auf die wir im Weiteren noch eingehen werden. Zunächst können wir aber festhalten, dass fast alle ProfessorInnen die Verbindung von Lehre und Forschung als wichtiges Qualitätsmerkmal betrachten. Dies wird nicht nur in Beschreibungen konkreter Lehrveranstaltungen oder -formate deutlich, sondern ebenso in Vergleichen zwischen universitärer und fachhochschulischer Lehre. So äußert sich ein jüngerer Strafrechtsprofessor folgendermaßen:

„Das ist ja tatsächlich so ein Punkt, seine Forschung dann auch in die Lehre einfließen lassen zu können. Also das ist eben in einem bestimmten Umfang einfach möglich. Insbesondere bei den Fortgeschrittenenveranstaltungen. Und das ist tatsächlich ein Punkt, den, glaube ich, Fachhochschulen nicht gleichermaßen leisten können." (JUR12)

Noch dezidierter hebt ein Professor für Zellbiologie die Relevanz einer Integration von Forschung und Lehre hervor, wenn er betont, dass Studierende

„,das Gefühl haben [müssen], ganz nah dran zu sein an den wichtigen, an den Wissenschaftsfronten, in Anführungsstrichen, das ist ein ganz, ganz wichtiger Aspekt, das schafft Motivation. Alles andere ist die Fortführung der Schule mit anderen Mitteln, und das wollen und das können wir nicht. Das sollen wir auch nicht. Ich glaube nicht, dass das der Auftrag ist.“ (BIO11)

Mit der Aussage, dass es sich ohne Forschungsbezug um die „Fortführung der Schule mit anderen Mitteln" handele, behauptet der Zellbiologe zum einen sein berufliches Selbstverständnis, kein bloßer „Lehrer“ zu sein. Zum anderen betont er aber gerade auch den Mehrwert für die Studierenden. Schließlich verknüpft er deren „Motivation“ mit der Möglichkeit, qua Lehre in Berührung mit 
den „Wissenschaftsfronten“ zu kommen. In ähnlicher Weise argumentiert ein Professor für Hochfrequenztechnik, wenn er hervorhebt, dass die Studierenden „unglaublich hungrig“ darauf seien, mehr über die aktuelle Forschungsarbeit „im Institut“ zu erfahren, anstatt „nur den trockenen Stoff“ vermittelt zu bekommen (TECH1).

In den zitierten Äußerungen spiegeln sich bereits unterschiedliche Deutungen einer Integration von Lehre und Forschung wider. Denn während der Strafrechtler darunter versteht, dass Forschungsinhalte ,in einem gewissen Umfang“ in Veranstaltungen ,einfließen“, gehen der Zellbiologe und der Hochfrequenztechniker stärker von dem Anspruch aus, Studierende unmittelbarer an Forschungsprozessen teilhaben zu lassen. Diese Unterschiede in den Deutungen lassen sich, wie wir im Folgenden noch genauer sehen werden, in erheblichem Maße mit den unterschiedlichen disziplinären Möglichkeiten der Integration von Forschung und Lehre erklären.

Doch nicht nur die Art des Einbezugs von Forschung in die Lehre, auch der Umfang, in dem das möglich ist, variiert disziplinspezifisch. So werden einem Forschungsbezug der Lehre im Strafrecht relativ enge Grenzen gesetzt. Wie wir bereits gezeigt haben, dominieren hier Vorlesungen und am Staatsexamen orientierte Lehrinhalte, die oftmals nur wenig mit den Forschungsschwerpunkten der ProfessorInnen zu tun haben. Dies bestätigt die folgende Äußerung eines Strafrechtsprofessors:

\begin{abstract}
„,[A]lso wir machen ja kaum Seminare, machen immer diese Vorlesungen und dann müssen wir Dinge abdecken, die eben im Richtergesetz stehen. [...] Wenn ich eine Vorlesung halte, gibt es da vielleicht so zu drei, vier Prozent Bereiche, zu denen ich auch mal was geschrieben oder länger intensiv nachgedacht habe, wie man es zum Forschen tut. [...] Es ist schon so, dass ich dann da ein bisschen mehr zu sage, als ich sagen würde, wenn ich da nichts zu geschrieben hätte. Aber es geht meistens nicht gut, weil man einfach zu viel weiß. [...] Zum Betrug habe ich sehr viel geschrieben, aber die [Studierenden, Anm. der AutorInnen] hören erstmals im dritten Semester: Was ist der Betrug? Was sind die Voraussetzungen? Und da brauche ich einfach schon die Zeit, vielleicht sind das drei, vier Doppelstunden im Semester, für die grundlegenden Dinge.“ (JUR6)
\end{abstract}

Der Strafrechtler betont vor allem die Hürden für einen stärkeren Forschungsbezug. Zum einen bestehe nur eine geringe Schnittmenge zwischen den vorgegebenen Inhalten und seinen Forschungsinteressen, zum anderen würde er die Studierenden durch eine inhaltliche Vertiefung tendenziell überfordern. Wenn es in Vorlesungen um eines seiner Forschungsthemen gehe, sage er zwar „ein 
bisschen mehr" dazu, letztlich sind Forschung und Lehre für ihn aber eher voneinander entkoppelte Bereiche.

Eine jüngere Fachkollegin geht hingegen weit stärker von dem Anspruch aus, ihre Forschungsinhalte in die Lehre zu integrieren:

„Die Lehre lebt von der Forschung. Ganz stark. [...] Dadurch, dass ich die Basics bringen möchte, kommt in der Lehre nicht so viel heraus, was ich dann in die Forschung einbringen möchte. Aber was ich gerade beforsche, das bringe ich sofort in die Lehre - indem ich ein Seminar mache zu dem Thema oder indem ich besonders gerne darüber rede, wenn es sich gerade anbietet. Also die Forschung prägt meine Lehre stark. Deswegen glaube ich auch, dass sozusagen ein Modell, wo man nur unterrichtet und nicht forscht, dass die Lehre armseliger wird, für beide Seiten. [...] Einfach nicht so engagiert, nicht so begeistert, nicht so durchdrungen, alles Mögliche. Ich glaube, dass die Kombination von Forschung und Lehre extrem wichtig ist. Die Lehre wird wirklich bereichert." (JUR3)

Aus Sicht der Strafrechtlerin ist die Verbindung zur Forschung eine wesentliche Voraussetzung wirklich ,guter' Lehre. Auch innerhalb einer Disziplin weisen die professoralen Ansprüche an die Lehre also durchaus ein Spektrum auf. Denn obwohl die Strafrechtlerin ebenfalls examensrelevante „Basics“ vermitteln muss, orientiert sie sich mehr als ihr Fachkollege an einer „Kombination von Forschung und Lehre“. Zugleich wird deutlich, dass die Strafrechtlerin primär von einer forschungsbasierten Lehre und nicht von Formen des forschenden Lernens ausgeht, ${ }^{5}$ bei denen sich die Studierenden selbst aktiv an eine wissenschaftliche Herangehensweise respektive an Forschungsprozesse annähern.

Bei ProfessorInnen, in deren Disziplinen die Lehre stärker von Seminaren oder Laborpraktika bestimmt wird, finden wir entsprechend erweiterte Ansprüche an eine Integration von Forschung und Lehre. Auf die Frage nach einem besonders positiven Beispiel beschreibt ein Literaturwissenschaftler etwa ein Seminar, in dem die Studierenden auch selbst Fragestellungen entwickeln sollten:

„Die werden nicht völlig in das Offene geschmissen, aber sie können das Offene gefahrlos ausprobieren, und am Ende muss dann ein Exposé vorliegen, also eine kleine Forschungsperspektive entwickelt sein [...].“ (LIT9)

\footnotetext{
${ }^{5}$ Zur Differenzierung von forschungsbasierter Lehre, forschungsorientierter Lehre und forschendem Lernen siehe Ludwig Huber (2014).
} 
Entscheidend ist für den Professor, dass die Studierenden sich hier als Forschende „,ausprobieren“ können und ihre eher passiv-rezipierende Rolle verlassen. In ähnlicher Weise beschreibt eine Zellbiologin Master-Kurse, die direkt im Labor stattfänden und den Teilnehmenden ein forschendes Lernen ermöglichten:

„Da können wir Schwerpunkte setzen auch forschungsnah, [...] und da haben wir sehr kleine Gruppen. Manchmal nur fünf Leute. [...] Ist natürlich fantastisch. Die können Sie hier ins Labor mit reinnehmen. Da brauchen Sie gar nicht in einen speziellen Kursraum gehen, sondern meine Doktoranden betreuen die dann direkt im Labor mit. Und dann können wir Forschungsfragen mit denen bearbeiten, wirklich ganz neue Sachen. Das macht schon Spaß.“ (BIO3)

Obwohl die Betreuung zu größeren Teilen von ihren DoktorandInnen übernommen werde, sei auch ihr eigener Kontakt zu den Studierenden enger. Diese brächten sich hier stärker als in anderen Veranstaltungsformaten ein:

„Da kommen manchmal interessante Sachen bei raus. Und weil die auch so spontane Fragen stellen, ((lacht)) manchmal völlig verrückte Sachen, macht man auch mal was anderes und sagt: ,Okay, wenn ihr das vorschlagt, machen wir das mal. “”

Die Professorin erlebt es als positiv, dass die Teilnehmenden eine wissenschaftliche Neugier entwickeln und Fragen oder Ideen äußern. Allerdings haben die von ihr beschriebenen Kurse einen zeitlich begrenzten Rahmen, in dem die Rollen zwischen Lehrender und Lernenden klar aufgeteilt bleiben. Ein Fachkollege aus der Zellbiologie bewertet das Lehrformat der Exkursion aus diesem Grund als noch positiver. Dort könne tatsächlich ein weniger hierarchischer und vor allem von Erkenntnisinteresse getriebener Forschungsprozess entstehen:

\begin{abstract}
„Da kommen in der Regel nur noch Leute rein, die Interesse haben, und das ist ein ganz anderes Niveau. Das macht richtig Spaß. Weil man merkt, wie engagiert die sind. Und wenn wir auf Exkursion sind außerhalb, dann haben die auch ihren ganzen Privatkram abgelegt. Das interessiert nicht mehr, die können nix mehr machen auf den Inseln. Vor allen Dingen sind wir alle zusammengerauft. Dann arbeitet man auch von morgens vom Frühstück bis abends. Selbst nach dem Abendbrot wird da noch ins Labor gegangen." (BIO2)
\end{abstract}

Der Zellbiologe beschreibt solche Exkursionen als besondere Höhepunkte seiner Lehrtätigkeit. Entscheidend hierfür ist, dass sich die Haltung der Studierenden verändert und diese sich nicht aus einer pflichtethischen, sondern einer intrinsischen Motivation engagieren. In ähnlicher Weise äußert sich auch 
ein Literaturwissenschaftler mit Blick auf Summer Schools, die er seit einiger Zeit außerhalb der Vorlesungszeiten durchführe. In deren Rahmen erlebe er Studierende, die sich ohne äußeren Druck ganz auf die Themen einließen:

„Da vergessen die oft mittags zu essen, arbeiten wirklich bis abends durch und dann fällt irgendjemandem ein: ,Komm, jetzt rufen wir einen Pizza-Service an. '((lacht)) Also das funktioniert auch. Und das können dieselben Studenten sein, die vorher wenig sichtbar sind." (LIT4)

Wir haben den Anspruch eines Forschungsbezugs der Lehre bis hierher vor allem anhand eines Spektrums positiver Beispiele dargestellt. Die Relevanz dieses Anspruchs für die berufliche Identität zeigt sich aber gerade auch, wenn ProfessorInnen dessen Gefährdung durch die „Bologna“-Reformen thematisieren. In diesem Kontext kommen vor allem zwei Kritikpunkte zur Sprache, die wir bereits angesprochen haben: eine Einschränkung der Lehrfreiheit und -qualität durch Modularisierung und inhaltliche Vorgaben sowie, alternativ oder zusätzlich, eine veränderte, nämlich extrinsischer geprägte Haltung der Studierenden.

Die schon mehrfach zitierte ältere Zellbiologieprofessorin sieht den Forschungsbezug ihrer Lehre durch Beides gefährdet. Im Bachelor sei sie mit einem wachsenden Anteil an Studierenden konfrontiert, denen es an einem wirklichen inhaltlichen Interesse mangele. Ansätze einer Integration von Forschungsbezügen müssten daher immer stärker didaktischen Fragen weichen, die sich „auch Lehrer in der Schule“ (BIO3) stellten. So gehe es nicht zuletzt darum, ein „Entertainment“ zu bieten, um auch an kurzfristigem Punkteerwerb orientierte Studierende „am Ball halten [zu] können“. Im Master seien die Studierenden zwar viel interessierter und hätten zudem „Spaß“ an forschungsorientierten Lehrformaten im Labor. Jedoch fehle durch das modularisierte BachelorStudium oftmals das „Riesenfundament“, welches nötig sei, um die komplexen Zusammenhänge wirklich „,zu verstehen“. Während ihr die Lehre vor „Bologna“ noch großen $\mathrm{Spaß}$ gemacht habe, müsse sie sich nun immer mehr um die eigene Motivation bemühen. Dabei gewinnen die positiven Ausnahmen unter den Studierenden an Bedeutung:

„Es gibt auch Leute, die kommen nach einer Vorlesung dann zu einem und fragen so ganz vorsichtig mal was und denken, man würde sie nicht kennen. Aber man sieht sofort die interessierten Studierenden, sofort. Auch in der Masse sieht man die. Die haben einfach irgendwie lebendigere Augen und gucken und passen auf. Die kann man sich dann schon rauspicken. Das ist schon befriedigend, dass es solche Leute gibt, sonst würde man keine Lust mehr haben.“ 
Angesichts der eher indifferent rezipierenden „Masse“ werden die interessierten Studierenden entscheidend dafür, nicht gänzlich die „Lust“ an der Lehre zu verlieren. Letztlich verschieben sich die Prioritäten der Professorin jedoch, indem sie ihren Fokus seit „Bologna“ stärker auf ihre Forschungsarbeit richtet:

„Die Forschung steht bei mir wirklich im Vordergrund. Das muss ich ganz ehrlich sagen. Also früher war das anders. Da wollte man noch eine tolle Vorlesung aufbauen. [...] Nee, also dass ich da jetzt noch mal große Veränderungen machen will, muss ich ganz ehrlich sagen, mache ich nicht mehr. Da konzentriere ich mich lieber auf die Forschung. Und wie gesagt, die Studenten, die interessiert sind, kriege ich ja rein. Also das schaffe ich immer noch, genug zu bekommen."

Sie stellt diese stärkere Gewichtung der Forschung somit als einen Schritt dar, der eigentlich in Widerspruch zu ihrem Lehrethos steht.

Die Zellbiologin verringert lediglich den Aufwand für die Vorbereitung ihrer Veranstaltungen. Einem älteren Politikwissenschaftler geht es hingegen weitergehend auch darum, seine formale Lehrverpflichtung „zu reduzieren“ (POL6). Die Motive dafür liegen vor allem in den Vorgaben für die verschiedenen Module. Während man vor „Bologna“ häufiger ,einen Forschungsakzent" habe setzen können, dominiere nun stärker die Wiederholung standardisierter Lehrinhalte:

„Mein subjektives Gefühl ist, ich werde immer stärker zu einem Lehrer, der wie alle anderen Lehrer an Gymnasien irgendwie so ein File oder so einen Aktenordner hat, da zieht man dann die Vorlesung raus, und ich sage immer, man lebt so aus der Büchse. Also, ich ziehe Vorlesungen durch, und früher haben wir halt Vorlesungen zu Themen gehalten und nicht diese einführenden Überschläge.“

Der Professor erwähnt zwar an anderer Stelle, dass im Master-Bereich noch gewisse Freiräume bestünden, um ,eigene[] Forschungsinteressen“ einbringen zu können. Aufs Ganze betrachtet seien die Möglichkeiten aber nur „sehr begrenzt“. Insofern fällt sein Gesamtfazit negativ aus: Aus seiner Sicht habe man durch die Reform - „künstlich oder ganz bewusst oder unbewusst“ - eine „Trennung von Forschung und Lehre hergestellt“. Die einstige Idee universitärer Lehre sei in der Folge ,degeneriert“.

Neben solchen eher resignativen Stimmen finden wir jedoch ebenso Beispiele für ProfessorInnen, die ihren Anspruch einer Integration von Forschung und Lehre gegenüber den neuen Strukturen behaupten wollen. So beschreibt es eine jüngere Politikwissenschaftlerin als durchaus „frustrierend“, dass Lehre und Forschung durch die vielen „Pflichtkurse“ seltener „Hand in Hand“ (POL7) 
gingen. Um auch weiterhin noch „Spaß“ an der Lehre zu haben, plane sie aber, zukünftig konsequenter von ihren eigenen Forschungsinteressen auszugehen:

„[F]ür mich funktioniert Lehre dann am besten, wenn ich sie möglichst eng an die Forschung anbinden kann, und ich habe mich erst gestern mit einer Mitarbeiterin unterhalten. [...] Und als wir uns dann auch darüber unterhalten haben, habe ich gesagt: ,Also ich mache das jetzt immer so: Ich biete ein Seminar ((lacht)) an, wo ich was wissen will. Und die Studierenden sind für mich Mittel zum Zweck.' Aber das funktioniert sehr gut, weil das dann oft eine sehr klare Linie hat, wo es hingeht und die Studierenden sich gefordert fühlen. Und dann funktioniert es eigentlich ideal.“

Auch für die Studierenden ergäben sich durch einen stärkeren Forschungsbezug Vorteile. Ein „Bologna“-kritischer Literaturwissenschaftler argumentiert ähnlich. So setze er sich „gerne“ (LIT8) über formale Vorgaben hinweg, um seinem Anspruch einer Integration von Forschung und Lehre besser gerecht werden zu können. Zugleich profitierten ebenso die Studierenden von den größeren Freiräumen, die er ihnen bei der Erfüllung ihrer Seminarleistungen lasse:

„,[U]nd ich sage den Leuten auch immer: ,Ihr müsst a) nicht über euer Referatsthema eine Hausarbeit schreiben und ihr müsst b) überhaupt nicht über einen Seminartext die Hausarbeit schreiben. Irgendwas, was im Umkreis ist, was thematisch gut dazu passt, ist mir genau so lieb.' Und dann kommen Leute manchmal mit völlig eigenen Vorstellungen, haben dann irgendwelche Texte entdeckt und meinen, damit kann man was machen, und das finde ich dann schon mal gut. Also dass man sich wirklich sozusagen in so einem Transfer vom Seminar auf andere Texte überlegt, was man hiermit jetzt machen kann. Da möchte ich eigentlich nicht drauf verzichten.“

Der Literaturwissenschaftler sieht sich also durch diejenigen Studierenden bestätigt, die einen kreativen und eigenständigen Zugang zu den Themen entwickeln, was er als einen entscheidenden Lernerfolg auf ihrer Seite ansieht.

ProfessorInnen gehen, so zeigt sich, mit wahrgenommenen Hürden bei der Integration von Forschung und Lehre unterschiedlich um. Während sich bei einigen, nicht zuletzt älteren ProfessorInnen ein partieller Rückzug aus der Lehre rekonstruieren lässt, finden wir ebenso Versuche, sich innerhalb der neuen Rahmenbedingungen von hinderlichen Vorgaben zu emanzipieren. Der Anspruch eines Forschungsbezuges ist indes über disziplinäre und generationale Unterschiede hinweg Teil der beruflichen Identität. Entsprechende - und sei es selbst erkämpfte - Handlungsspielräume werden insofern von vielen ProfessorInnen als Voraussetzung dafür gesehen, als Lehrende motiviert zu bleiben. 
Lehrziele zwischen wissenschaftlicher Expertise, klassischem Bildungsideal und Beschäftigungsfähigkeit

Gerade angesichts der „Bologna“-Reformen stellt sich die Frage, welche Formen der Responsivität sich in Bezug auf die hochschulpolitische Erwartung zeigen, die universitäre Lehre stärker an der Beschäftigungsfähigkeit der Studierenden auszurichten. Zwar werden auch im Strafrecht die Bemühungen um eine praktischere Ausrichtung des Studiums sehr unterschiedlich gedeutet, da die Lehre und das Selbstverständnis der Lehrenden dort aber ohnehin viel stärker durch das Ziel der Examens- und Berufsvorbereitung bestimmt sind, bleibt das Strafrecht mit Blick auf die mit der „Bologna“-Reform angeschobene Forderung einer verbesserten „Employability“ hier ausgeklammert.

Ausgehend von dem wissenschaftlichen Anspruch an die Lehre, der sich in vielen Interviews zeigte, könnte man aufseiten der ProfessorInnen eine überwiegend ablehnende Haltung gegenüber dieser Erwartung vermuten. Die kritische Äußerung einer Professorin für Neuere Deutsche Literatur scheint diese Annahme zu bestätigen:

„Wenn wir umstellen von Forschung auf arbeitsmarktfähige Kompetenzen, dann ist es natürlich eben nicht mehr der Raum des Denkens, sondern sozusagen des gezielten Schulens, und damit ändert sich natürlich auch sozusagen das Berufsbild der Professorin.“(LIT13)

Die Literaturwissenschaftlerin deutet das forcierte Lehrziel ,arbeitsmarktfähige[r] Kompetenzen“ im Sinne einer „Landnahme“ (Dörre 2013), im Zuge derer entscheidende Freiheitsgrade verloren gingen. So werde der universitäre „Raum des Denkens“ letztlich zu einem Raum des „gezielten Schulens“. Nicht wenige der Professorinnen und Professoren setzen sich jedoch differenzierter mit der Frage auseinander, inwieweit die eigene Lehre auch einen Beitrag für die berufliche Zukunft der Studierenden - in und außerhalb der Wissenschaft - leisten kann bzw. sollte.

In den Natur- und Ingenieurwissenschaften findet diese Auseinandersetzung schon deshalb statt, weil dort das Ziel, Studierende qua Lehre für die Forschung zu begeistern und wissenschaftlichen Nachwuchs zu rekrutieren, eine besonders wichtige Rolle spielt. Die ältere Professorin der Zellbiologie beschreibt den aus ihrer Sicht idealtypischen Weg folgendermaßen:

„Also das Beste ist wirklich, im ersten Semester einer, der so Sie anguckt strahlend, ja? ((lacht)) Dem so einen HiWi-Posten zu geben, der macht ein bisschen Arbeiten hier für uns, und den bauen Sie jetzt richtig auf. Der lernt das Labor von der Pieke 
auf, der weiß alles, wenn der nachher hier ist und seine Masterarbeit macht. Dann kennt der alles schon hier. Der braucht sich dann hier nicht neu orientieren, sondern fängt sofort an. Dann kommt auch was bei raus.“ (BIO3)

Die Zellbiologin zielt auf einen umfänglicheren Sozialisationsprozess ab und spricht auch an anderen Stellen davon, die motivierten Studierenden allmählich „,aufzubauen“, damit diese die Denk- und Herangehensweisen kennen lernten, die die Forschungsarbeit vor Ort prägen:

„[D]ie Denkweisen müssen ja auch stimmen, man muss miteinander reden können. Manche Leute denken ja in ganz anderen Mustern als man selbst. Also die müssen sich ja aufeinander eingespielt haben, die Leute, die nachher eine Promotion hier machen. Die müssen wissen, wie ich denke. Sonst läuft das ja auch nicht. ((lacht))“

Das Motiv, über die Lehre wissenschaftlichen Nachwuchs zu gewinnen, spielt ebenso für einen jüngeren Fachkollegen eine wichtige Rolle. So sieht der Zellbiologieprofessor in Master-Studierenden nicht zuletzt „das Kapital“, aus dem man für die Forschung ,schöpfen“ (BIO11) könne, und in Veranstaltungsformaten wie Praktika eine Art Testlauf für die Wissenschaft.

Wir finden jedoch ebenso ProfessorInnen, die sich stärker mit der angemessenen Gewichtung von Forschungs- und Praxisorientierung auseinandersetzen und bei ihren Lehrzielen explizit den außeruniversitären Arbeitsmarkt in den Blick nehmen. So grenzt sich ein älterer Politikwissenschaftler von KollegInnen ab, die der Devise „,mir sind die Studierenden eigentlich egal“ (POL6) folgten und selbstgewiss behaupteten:

„[I]ch weiß, dass die viel mehr davon haben, wenn sie jetzt das machen, was ich mache, und dadurch mehr Theorie und forschungsorientierte Ausbildung bekommen."

Er selbst nehme gegenüber dem gewachsenen Wunsch der Studierenden nach Praxisbezügen und ihrer Frage, ,was sie mit dem Zeug da draußen anfangen“ könnten, keine solche abwehrende Haltung ein. Als Lehrender habe er sich auch auf die Situation und die „Bedürfnisse“ der Lernenden ,,einzustellen“:

,[I]ch verstehe die ja. Und nicht jeder kann in die Forschung und nicht jeder soll in die Forschung. Deswegen würde ich eher gezielt Kurse anbieten für Leute, die wirklich in die Forschung wollen. Ansonsten, ich meine, was soll man mit den ganzen Leuten in der Forschung? Die müssen ja irgendwo da raus auf den Arbeitsmarkt.“ 
Neben der Vermittlung eines für den Arbeitsmarkt wichtigen „Handwerkszeugs" versucht der Politikwissenschaftler, in möglichst vielen Veranstaltungen das ,Theorie/Praxis-Verhältnis“ zu berücksichtigen. Dies sei ihm bereits bei der inhaltlichen Gestaltung seiner Vorlesungen ,wichtig“, lasse sich in Formaten wie Praxis-Seminaren jedoch noch anschaulicher und berufsfeldspezifischer realisieren:

\begin{abstract}
„Also bei 14 Semesterwochenstunden lesen wir sieben Semesterwochenstunden Texte, also zu politischer Praxis und wo sich Politologen dann im Berufsfeld wiederfinden. In den anderen sieben Veranstaltungen lade ich Praktiker ein -also Politologen, die da draußen in den Institutionen arbeiten, in Gewerkschaften, in Ministerien, in Parteien als Politologen, die dort Jobs gefunden haben und die dann reinkommen und erzählen, a) was sie da machen, b), was sie aus dem Studium mitbringen oder mitgebracht haben, was man aus dem Studium überhaupt benutzen kann für diese Art von Jobs und so.“
\end{abstract}

Für den Professor verbindet sich mit dieser Neuausrichtung seiner Lehre allerdings auch eine Ambivalenz. Während er auf der einen Seite einem Verantwortungsgefühl für die Studierenden folgt und ihm solche Veranstaltungen durchaus auch „Spaß“ machten, bedauert er auf der anderen Seite, dass nur noch wenige seiner Studierenden eine Affinität zur Forschung zeigten. Letztlich bleibt für ihn der eigene Anspruch einer Integration von Forschung und Lehre zu sehr auf der Strecke.

Eine Ambivalenz spiegelt sich auch in den Äußerungen eines jüngeren Fachkollegen wider. Ursache ist bei ihm vor allem seine Unsicherheit über die „richtige“ (POL1) Verbindung von forschungs- und praxisorientierten Anteilen. So sucht der erst vor wenigen Jahren auf seine Professur berufene Politikwissenschaftler noch nach der geeigneten „Strategie“, um den Studierenden beide Wege - in die Wissenschaft, aber auch in andere Berufsfelder - zu eröffnen:

„Ich glaube auch, dass das wichtig ist, den Leuten diese wissenschaftliche Zugangsweise und auch wissenschaftliches Handwerkszeug mitzugeben, auch wenn sie aus der Uni rausgehen. [...] Das ist aber eine Sache, das sozusagen auf einem allgemeinen Niveau zu machen. Das ist nochmal eine andere Sache, das hier sozusagen explizit für einen wissenschaftlichen Arbeitsmarkt zu trennen. Ich glaub, das ist so ein bisschen die Unterscheidung. Ich versuch im Moment immer, bisschen beides zu machen. Ich weiß aber nicht, ob das jetzt eine schlaue Strategie ist ((lacht)) oder keine schlaue Strategie ist. Es ist ein Spagat. Also ich will es den Leuten nicht verbauen, [...] man will ja auf der anderen Seite auch keine FH-Ausbildung machen." 
Eine solche Zielkonkurrenz sieht ein Nachrichtentechniker hingegen kaum. Er hebt vielmehr hervor, dass in seiner Disziplin hinsichtlich der notwendigen Kompetenzen eine große Schnittmenge zwischen Wissenschaft und Industrie bestehe. In beiden Bereichen seien soft skills wie Selbstorganisation, Zeitmanagement, das Arbeiten im Team sowie eine lösungsorientierte Vorgehensweise erforderlich. Aus diesem Grund ist es ihm auch wichtig, diese Kompetenzen durch seine Lehre zu fördern:

\begin{abstract}
„Mir geht es darum, dass sie selber aktiv das tun, weil -ich sage mal so- einem Stoff zuzuhören in einer Vorlesung ist eine Sache, es zu verstehen ist schon eine kompliziertere Sache, es selber aber anschließend anwenden und diese Probleme zu lösen, das ist das Schwierigste. Das sollen sie aber lernen, weil das brauchen sie anschließend im Berufsleben und in der Prüfung natürlich genauso. Deswegen gestalten wir diese Übungen eher als aktive Übungen, wo die Studenten direkt selber aktiv sind, und dann in der Gruppe, damit sie untereinander ein bisschen sich helfen und auch mal lernen, in der Gruppe gemeinsam, ein gemeinsames Problem mal in der Kürze der Zeit lösen zu können. Da liegt mir viel dran, und entsprechend haben wir auch in den letzten drei Jahren oder so angefangen, das Konzept [der Übungen, Anm. der AutorInnen] mal umzustellen [...].“ (TECH2)
\end{abstract}

Der Nachrichtentechniker deutet solche Umstellungen nicht im Sinne einer ambivalenten Anpassung. Die Orientierung der Lehre an späteren beruflichen Herausforderungen ist für ihn ohnehin ein Selbstanspruch, an dem er die Qualität seiner Lehre misst. Dies lässt sich mit dem stärkeren Anwendungsbezug seiner Fachdisziplin, aber ebenso mit seiner beruflichen Sozialisation erklären. Denn er wechselte in seinem beruflichen Werdegang zwischen Fachhochschule, Industrie und Universität und verteidigt weniger als andere KollegInnen die besondere Autonomie universitärer Lehre.

Die hochschulpolitisch forcierte „Employability“-Norm wird also keineswegs durchgängig abgewehrt oder ausgeblendet. Wir finden in den Interviews vielmehr unterschiedliche Formen der Responsivität, die sich zwischen den Polen einer dezidierten Vereinnahmungskritik und der problemlosen Aneignung eines stärkeren Arbeitsmarktbezugs der Lehre bewegen. Mit dem Beispiel des älteren Politikwissenschaftlers (POL6) konnten wir zudem exemplarisch zeigen, dass sich ProfessorInnen in ihrer Lehre durchaus auch zulasten eigener wissenschaftlicher Ansprüche an den beruflichen Optionen der Studierenden orientieren. Das teilweise in journalistischen Diskursen gezeichnete Bild von UniversitätsprofessorInnen, die ihre Lehre unreflektiert, wenn nicht gar verantwortungslos, den eigenen Forschungsinteressen unterwerfen, haben wir bei unseren Fällen nicht vorgefunden. 
Zum Lehrethos nicht weniger ProfessorInnen gehört darüber hinaus aber ebenso der Anspruch, Haltungen und Fähigkeiten zu vermitteln, die eher dem nicht zweckgebundenen erweiterten humanistischen Bildungsbegriff entsprechen. Besonders ausführlich geht ein älterer Literaturwissenschaftler auf das Lehrziel einer umfänglicheren „Charakterausbildung“ der Studierenden ein, welches für ihn eindeutig „,vorrangig“ (LIT1) sei. ${ }^{6}$ Er führt dieses Lehrziel so weiter aus:

\begin{abstract}
„Ich glaube, dass unser Fach ein ästhetisches Fach ist und dass es zunächst darum geht, Kunstwerke in ihrer Kunstartigkeit zu verstehen, so wie das in der Malerei oder in der Musikwissenschaft auch ist. Aber gerade in der Literaturwissenschaft geht es auch darum, durch das Verstehenlernen, das systematisch geleitete, methodisch reflektierte Verstehen von Texten, Empathie zu entwickeln und sich in andere Menschen, andere Auffassungen, andere Kulturen, andere Zeitalter versetzen zu können, soweit das eben möglich ist, und auch die Distanz zwischen dieser Kultur dort und meinem Dasein hier mit zu reflektieren. Das ist auch das, was ich antworten würde auf die Frage, warum gesellschaftlich eigentlich ein Fach wie Literaturwissenschaft überhaupt sinnvoll ist über das rein Museale hinaus oder das rein Ästhetisch-Genießerische hinaus. Und da würde ich sagen: Wir sind eine Schule für methodisch reflektierte, angeleitete Empathie. [...] Also das ist schon der alte Humboldtsche Bildungsbegriff.“
\end{abstract}

Idealerweise verbindet sich für den Literaturwissenschaftler beides miteinander: die Fähigkeit, literarische Texte durch eine systematische Analyse in ihrer „Kunstartigkeit zu verstehen“, und die Entwicklung einer Empathie, die möglichst zu einer generalisierten Haltung wird.

Andere ProfessorInnen nehmen zwar keinen expliziten Bezug auf das klassische Bildungsideal von Humboldt. Gleichwohl äußern auch sie Lehrziele, die über einen enggefassten Kompetenzbegriff hinausgehen. $\mathrm{Zu}$ diesen Lehrzielen zählt die Befähigung zur kritischen Reflexion. Eine jüngere Professorin für Politikwissenschaften konkretisiert dieses Ziel etwa anhand ihres Anspruchs, den Studierenden eine Distanz gegenüber medialen und politischen Diskursen zu vermitteln. So habe sie die „Attentate von Charlie Hebdo“ zum Anlass genommen, um in einer „Extra-Vorlesung“ die Presseberichterstattung „über den Islam“ (POL7) zu rekonstruieren. In diesem Fall hätten die Studierenden zwar vor allem nach der Klausurrelevanz gefragt und sich nur bedingt auf die avisierte

\footnotetext{
${ }^{6}$ Es handelt sich um jenen Professor, der sich - siehe Kapitel 3.1 - dezidiert gegen die „Bologna“-Reformen gewendet und diese als massiven Einschnitt in die Lehr- und Lernfreiheit gedeutet hatte.
} 
Perspektive eingelassen. Bei anderen Veranstaltungen mache die Politikwissenschaftlerin jedoch auch positivere Erfahrungen:

\begin{abstract}
„Wir haben immer wieder Gastvorträge über Erasmus Staff Exchange, wo wir ja wirklich Leute aus ganz anderen Ländern und Universitäten bekommen, und wenn die da einen Vortrag halten und das ist ein politischer Vortrag, dann fragen mich inzwischen meine Studierenden: ,Aber das war ja schon irgendwie ein recht emotionaler Vortrag'. ((lacht)) Dann bin ich immer ganz stolz, weil ich denke, ,okay, es kommt was an'. Die erkennen das auch, die nehmen das nicht für bare Münze, sondern erkennen, das ist jetzt eine recht subjektiv gefärbte Position. Also von daher, denke ich mal, scheint es schon zu funktionieren, so wie ich das angehe. Und sie erkennen da, glaube ich, einen Wert drin.“
\end{abstract}

Das Lehrziel, Studierende zu einer kritischen Rezeption zu befähigen, wird ebenso von einigen der StrafrechtsprofessorInnen geteilt. So ist es einem Strafrechtler wichtig, neben dem Fachwissen auch eine Metaperspektive auf die geltenden Gesetze zu vermitteln. Er mache schon den StudienanfängerInnen deutlich,

„dass es eben letztlich um Offenheit geht im Nachdenken, ob der Stoff, die Regeln, die Rechtsregeln richtig sind oder ob die Rechtsprechung zustimmungswürdig ist oder ob man es anders besser machen sollte und so weiter. Dass das eine wichtige Dimension ist, wenn man Lehre an einer Universität betrachtet. Und jetzt nicht nur zum schnellstmöglichen Berufseinstieg." (JUR1)

Ein weiterer Strafrechtler verfolgt ebenso das Ziel, mit seiner Lehre zu einer solchen Reflexion beizutragen. Die Möglichkeit sieht er hierfür insbesondere in der Kriminologie, in der er neben dem regulären Strafrecht Veranstaltungen anbietet:

„[I]ch genieße das schon, dass ich diese atypischen Strafrechts-Veranstaltungen machen kann, die Kriminologie, und sozusagen eher die strafrechtskritischen Fächer betreiben und da so eine wirklichkeitsbezogene Perspektive hineinbringen kann. Und das ist dann weniger strafrechtsdogmatisch angelegt, wie die Studierenden das sozusagen aus den normalen strafrechtlichen Veranstaltungen kennen, sondern eher sozusagen geprägt durch einen sozialwissenschaftlichen Blick auf das Strafrecht und damit auch einen viel kritischeren Blick auf das Strafrecht.“ (JUR10)

Beide Professoren eint der Anspruch, den Studierenden den gesellschaftlichen Kontext und die Veränderbarkeit geltender Gesetze sichtbar zu machen.

Bei einigen der ZellbiologInnen finden wir hingegen das Ziel, durch die Bezugnahme auf globale Herausforderungen zu einem Problembewusstsein der 
Studierenden beizutragen. Die ältere Zellbiologin beschreibt dieses Lehrziel eher als ein nicht so gut ankommendes. Wenn sie in Veranstaltungen etwa die Frage aufwerfe, wie aus dem Wissen über Pflanzenzellen ein Beitrag zur Welternährung geleistet werden könnte, treffe sie nur selten auf Interesse:

„[S]o einen globalen Blickwinkel da reinzubringen, das können Sie versuchen, aber da werden Sie höchstens mitleidig angeguckt: Och, die immer mit ihrem Moralischen. Das kann man mal sagen, aber wenn man das öfter macht, ich glaube, da hören die nicht mehr zu. Also der Welthunger ist einfach für die nichts so direkt Fassbares.“(BIO3)

Weniger resigniert äußert sich ein Fachkollege, der bei seinen Studierenden ein Bewusstsein für den Klimawandel und einen verantwortlichen Umgang mit Ressourcen stärken möchte:

\begin{abstract}
„Mir ist Umweltschutz wichtig. Ich sage dann immer, wer, wenn nicht wir, wer denn sonst? [...] Aber [...] wir laufen da auf den Abgrund zu. Dadurch, dass die Weltbevölkerung ansteigt und die Dritte Welt, die wollen ja nun auch mehr Energie verbrauchen, die wollen ja auch nicht auf so einem niedrigen Standard bleiben, und das muss ja irgendwo bereitgestellt werden. Es geht auf Belastung der Umwelt. Und das ist das, was man hier auch lehren kann und beibringen und hoffen kann, das Bewusstsein zu schüren, dass die Leute das wahrnehmen.“(BIO2)
\end{abstract}

Bis hierhin können wir also ein Spektrum an Deutungen guter Lehre und damit verbunden auch der wahrgenommenen Möglichkeiten und Grenzen der Umsetzung zentraler Ansprüche an die Lehre aufzeigen.

\title{
3.3 Gute' Forschung
}

Die zentrale Bedeutung der Forschung für die berufliche Identität der interviewten ProfessorInnen ist schon deshalb naheliegend, weil die Karrierechancen und die Reputation innerhalb der Scientific Community weiterhin wesentlich von den Forschungsleistungen abhängen und eine starke Orientierung an der Forschung inhärenter Teil der beruflichen Sozialisation ist. ${ }^{7}$ Unsere Interviews zeigen jedoch darüber hinaus, dass der eigene Bezug auf die wissen-

\footnotetext{
${ }^{7}$ Dies zeigen auch repräsentative empirische Untersuchungen zum Berufsbild der HochschullehrerInnen (Enders 1998; Teichler 2014).
} 
schaftliche Tätigkeit von den meisten ProfessorInnen in starkem Maße über ihre intrinsische Motivation definiert und beschrieben wird. So begründet ein Strafrechtler die große subjektive Bedeutung der Forschung etwa mit dem „Gefühl der Befriedigung“, das sich für ihn mit der Erfahrung verbinde, qua wissenschaftlicher Praxis den eigenen „Horizont weiterentwickeln zu können“:

„Mich befriedigt sehr auch, [...] anderen Menschen dazu zu verhelfen, dass sie etwas lernen, aber ich bleibe natürlich stehen. Und mein eigenes, sagen wir mal originäres, existenzielles, wenn Sie so wollen Interesse, das mich mal überhaupt in die Wissenschaft geführt hat, war, selber Erkenntnisse zu gewinnen.“ (JUR1)

Der Strafrechtler geht von einer biografischen Kontinuität seiner Suche nach neuen Denkweisen und Erkenntnissen aus. So habe er es bereits ,,von Kindesbeinen an“ als ein ,großes Faszinosum“ erlebt, in neue „Gedankenwelten einzutauchen“, und insofern die entsprechenden habituellen Dispositionen für seinen späteren Berufsweg ,schon vorher mitgebracht“. Sein akademischer Lehrer an der Universität, der eine „,charismatische Persönlichkeit“ gewesen sei, habe ihn dann, ,ins wissenschaftliche Denken, in die wissenschaftliche Arbeit überhaupt [...] hineingesogen" und seiner vorhandenen Neigung eine klarere Richtung gegeben.

Der Strafrechtler beschreibt seine berufliche Identität als Wissenschaftler somit vor allem über eine starke intrinsische Motivation, die immer schon in ihm vorhanden gewesen sei. Er folgt einer Selbstdeutung, die Hildegard Matthies (2015, S. 191/192) dem Typus der Karriere durch Selbstentfaltung zuordnet, der sich dadurch auszeichnet, die wissenschaftliche Tätigkeit als ,etwas genuin Eigenes zu begreifen“ und „Person und Sache“ als eine Art „untrennbare Einheit“ zu sehen. Auch ein Professor für Neuere Deutsche Literatur folgt einer solchen Selbstdeutung, wenn er davon spricht, dass die Praxis des Forschens mit den „,ersten Gedanken“ des Tages beginne und im Grunde seine „Lebensform“ (LIT1) sei. Entsprechend deutlich grenzt er sie von einer regulären, örtlich und zeitlich gebundenen Erwerbsarbeit ab: ${ }^{8}$

„Es ist nicht so, dass ich denke: ,Jetzt gehe ich zur Arbeit und forsche‘, sondern ich stehe morgens auf, und mit dem ersten Gedanken fang ich an zu forschen, mache mir Notizen und das läuft irgendwie immer so mit. Das ist mein Hobby sozusagen.“

\footnotetext{
8 Ähnliche Beschreibungen einer „Wissenschaft als Lebensform“ finden sich bei Jürgen Mittelstraß (1982, 2006).
} 
Vergleichbare Äußerungen, in denen der eigene Bezug auf die Forschung als völlig unabhängig von Rollenerwartungen oder der wissenschaftlichen Reputationsordnung gerahmt wird, finden sich zwar eher selten. Unsere Interviews zeigen jedoch durchgängig, dass sich die Bedeutung der Forschung nicht auf karrierestrategische Motive oder den - wenn auch nicht unbedeutenden Wunsch nach fachkollegialer Anerkennung reduzieren lässt. Dies verdeutlichen auch Äußerungen von ProfessorInnen, die sich nach einer erfolgreichen beruflichen Phase in der Wirtschaft um eine Rückkehr an die Universität bemühten. Ein Nachrichtentechniker begründet etwa seinen Wechsel aus der Industrie vor allem mit der größeren Freiheit, eigenen Forschungsinteressen folgen zu können:

„Viele Forschungsthemen, die vielleicht mich persönlich interessiert hätten, waren nicht umsetzbar. Wenn man jetzt dagegen als Professor agiert, dann hat man natürlich wieder die Freiheit, ich sage mal so, einfach das zu tun, was man gerne möchte, und wo man auch langfristig natürlich irgendwo unabhängig ist, und ob ich jetzt damit eine Marktmarge erziele oder nicht, mich interessiert die Forschung selber, die Technik selber.“ (TECH2)

Ähnlich wie bei den Deutungen ,guter` Lehre sollen im Folgenden neben Gemeinsamkeiten auch Unterschiede in den professoralen Ansprüchen an die Forschungsarbeit verdeutlicht werden. Diese Unterschiede betreffen die für das Selbstverständnis wichtigen Fragen des Verhältnisses von Individual- und Teamforschung sowie des Verhältnisses wissenschaftlicher Bezüge auf der einen, außerwissenschaftlicher Bezüge zu anderen gesellschaftlichen Sphären auf der anderen Seite. Zuvor werden wir jedoch die Bedeutung des klassischen Motivs der Curiositas nachzeichnen, das auf einen Erkenntnisgewinn jenseits des bekannten Wissens abzielt und sich bereits in einigen der bisher zitierten Äußerungen widerspiegelte.

\section{Curiositas}

Das Curiositas-Motiv spielt in fast allen der Interviews eine wichtige Rolle. Sobald die Sprache auf besonders positive Forschungserfahrungen kommt, werden von den ProfessorInnen vor allem Beispiele für Projekte oder Publikationen geschildert, mit denen sie einen ganz neuen wissenschaftlichen Beitrag leisten konnten.

Die bereits zitierte ältere Zellbiologieprofessorin beschreibt eine für sie besonders sinnstiftende Forschungsphase folgendermaßen:

„Aber so vor zehn Jahren gab es dann etwas, da habe ich gewusst, ,okay, das ist ein Ergebnis, das steht nicht im Lehrbuch, das ist völlig neu'. Da kribbelt es einen, 
dass man so was findet plötzlich, dass man nicht nur immer was wiederholt und vielleicht aus einem anderen Blickwinkel noch mal das Gleiche zeigt. Das geht lange Zeit so, und plötzlich hatte ich was ganz Neues in der Hand.“ (BIO3)

Nach einer längeren Durststrecke „eine völlig neue Entdeckung“ zu machen, wird von der Zellbiologin als eine Art Schlüsselerlebnis beschrieben. Ihre wissenschaftliche Arbeit habe dadurch „ein anderes Niveau“ erreicht. ${ }^{9}$ Zudem sei es „unglaublich“, wie motivierend die Ergebnisse seien. Es verstärkte das Erfolgserlebnis noch, dass sie sich erst gegen Vorbehalte innerhalb der Scientific Community durchsetzen musste - ,weil das gegen alles geht, was in Büchern steht":

„[E]s breitet sich dann so aus. Es kommen immer mehr dazu, die das toll finden. Das macht dann schon Spaß. Aber erstmal muss man eine Hürde nehmen. Also am Anfang muss man, darf man nicht aufgeben, wenn sie einem nicht glauben.“

Zum einen unterscheidet die Zellbiologin zwischen einer Art Standardforschung, die sich auf bereits bekannten Pfaden bewegt, und einer wirklich innovativen Forschung höheren Niveaus, mit der etablierte Annahmen widerlegt werden. Zum anderen betont sie die Relevanz des hierfür nötigen Forschergeistes, zu dem eben auch die Bereitschaft gehöre, sich an „Hürden“ zu bewähren.

Die Forschungspraktiken und Wege zu neuen Erkenntnissen werden stark von den jeweiligen disziplinären Eigenschaften bestimmt. So findet sich die Rede überraschender Entdeckungen im Zuge ergebnisoffener Experimente vor allem in den Interviews mit NaturwissenschaftlerInnen. Der Anspruch, in der Forschung Neuland zu betreten, besteht jedoch gleichermaßen in den sozial- und geisteswissenschaftlichen Disziplinen. So setzt ein Strafrechtsprofessor seine Forschung explizit mit einer „Entdeckungsreise“ gleich, auf der er sich auf unterschiedliche Themen einlasse und „notorisch neugierig“ (JUR1) bleibe. In ähnlicher Weise betont ein Literaturwissenschaftler die Offenheit für Unentdecktes als kontinuierliches Merkmal seiner Forschung. Nach der Arbeit mit „,öllig abgedrehte[n]“ und nahezu unbekannten Quellen zu Beginn seiner Karriere interessiere er sich derzeit für den Bereich der Digital Humanities. Durch die Anwendung computer-

\footnotetext{
${ }^{9}$ Ähnliche Beobachtungen finden sich bei Andreas Franzmann (2012), der der Bewältigung sich selbst auferlegter Krisen im Forschungsprozess eine für die Ausbildung des ForscherInnenhabitus essentielle Rolle beimisst.
} 
gestützter Verfahren erfahre er eine neue Form des Erkenntnisgewinns, die eher dem experimentellen Charakter naturwissenschaftlicher Forschung entspreche:

„, [A]lso wenn dann was funktioniert, da gibt es schon so einen Moment - da geht es nicht nur mir so ((lacht)), sondern auch meinen Kollegen: Da springen wir vom Stuhl auf: ,Wah, das hat geklappt!‘ Also so stelle ich mir das auch im Labor vor, wenn die dann jahrelang in irgendeine Richtung gearbeitet haben, und dann klappt es. Das ist dann schon so ein Heureka-Moment." (LIT4)

Der Germanistikprofessor erlebt jedoch ebenso „ganz viele Vorträge“ und „Aufsätze" als sinnstiftend, mit denen er sich stärker auf dem Terrain typischer geisteswissenschaftlicher Forschung bewegt. Wichtiger Anspruch ist für ihn, etwas Neues beizutragen - beispielsweise selbst zu klassischen Autoren wie „Goethe und Schiller“ etwas herauszuarbeiten, „was einfach tatsächlich noch niemand gesehen“ habe. „Nix Tolles“ seien hingegen Arbeiten, bei denen „man schon vorher ungefähr" wisse, ,was raus" komme:

„Das macht dann keinen Spaß, das spürt man ja, also spüre ich dann sofort, dass es irgendwie kein Erkenntnisfortschritt ist. Das hat keine Bedeutung letztendlich für einen, für mich selbst wie für andere auch.“

Der fehlende Erkenntnisfortschritt lässt solche wissenschaftlichen Arbeiten nicht nur für die Scientific Community, sondern gerade auch für ihn selbst bedeutungslos werden. Damit deutet der Literaturwissenschaftler, gute' Forschung in ähnlicher Weise wie die eingangs zu Wort gekommene Zellbiologin.

Wir können soweit festhalten, dass der Anspruch, etwas Innovatives beizutragen, und die hierfür notwendige Haltung der Curiositas als immanente Bedingungen wissenschaftlichen Fortschritts zentral für die berufliche Identität der interviewten ProfessorInnen sind.

\section{Forschung als individueller oder vergemeinschafteter Prozess}

Daran anschließend möchten wir nun näher betrachten, ob Forschung als vor allem individuelles oder als stärker vergemeinschaftetes Erkenntnisstreben gedeutet wird. Diese Frage ist auch mit Blick auf den Bedeutungsgewinn von Projekt- und Verbundforschung interessant, der sich in der Forschungsförderung und in universitären Leistungsbewertungen widerspiegelt. Dabei differenzieren wir zwischen der Bearbeitung von Fragestellungen mit einem Team von NachwuchswissenschaftlerInnen, die zumeist dem eigenen Lehrstuhl oder der eigenen Arbeitsgruppe angehören, und der Einbindung in inter- oder intra- 
organisationale Kooperationen mit FachkollegInnen oder ProfessorInnen anderer Disziplinen.

Mit Blick auf die erste Variante zeigen sich klare disziplinäre Unterschiede. Denn anders als in der Neueren Deutschen Literatur oder im Strafrecht setzen die ProfessorInnen aus den Natur- und Ingenieurwissenschaften die Arbeit im Team als mehr oder weniger selbstverständlich voraus. Bereits in unseren Ausführungen zur Lehre spiegelte sich wider, dass sich ProfessorInnen aus den entsprechenden Disziplinen darum bemühen, gute Studierende möglichst früh in die praktische Arbeit am Lehrstuhl einzubinden. Die enge Zusammenarbeit wird in den Interviews nicht nur als notwendige Bedingung, guter' Forschung, sondern auch als besonders positiver Aspekt der Arbeit herausgestellt. Entscheidend für den „Spaß an den Naturwissenschaften“ ist für eine Zellbiologieprofessorin etwa die Haltung, dass ,alle an einem Strang ziehen“ (BIO3). Bereits als Nachwuchswissenschaftlerin erlebte sie die Arbeit im Team als besonders inspirierend. Die „Kreativität“, die sich damals im Forschungsinstitut mit anderen BiologInnen habe ,entfalte[n]“ können, wurde für sie zum entscheidenden Motiv, sich für eine wissenschaftliche Laufbahn zu entscheiden.

Mit der Handlungskonstellation eines Teams verbinden sich für die ProfessorInnen zugleich spezifische Anforderungen. Als ProjektleiterInnen bestimmen sie die Forschungsfragen und sind in der Umsetzung des Forschungsprogramms für die Gesamtkoordination und die Betreuung ihrer Mitarbeitenden, insbesondere derjenigen in Qualifizierungsphasen, verantwortlich. Dies zeigt sich etwa in den Äußerungen eines Nachrichtentechnikers, der seine Aufgaben nicht zuletzt in Motivation und Teambildung sieht:

\footnotetext{
„Was wir dann auch versuchen, ist eben, dieser Spirit muss auch unter den Mitarbeitern entstehen. [...] Also ich kann ja die Umgebung bereitstellen, aber der Geist, der wird ja von jedem Mitarbeiter hier mitgeprägt. Ich versuch eben immer, zu sagen, wie es sein, was für'n Spirit herrschen soll.“ (TECH3)
}

Der gemeinsame „Geist“ ist ein Erfolgsparameter, der gegenüber der materiellen und personellen Ausstattung nicht geringgeschätzt werden darf. Ähnlich wie im Fall der Zellbiologin zeigt sich hier die Relevanz der beruflichen Sozialisation. Es sind vor allem Erfahrungen aus Forschungsabteilungen verschiedener Unternehmen, die die Ansprüche des Nachrichtentechnikers an die eigene Rolle prägen. So orientiert er sich an dem Ideal einer Mitarbeiterführung, die ,relativ viele Freiheiten“ lasse und auf „Vertrauen“ anstatt auf Kontrolle und Druck basiere: 
„Druck ausüben führt nicht dazu, dass die Arbeit besser wird. Natürlich muss eine gewisse Form von Druck ausgeführt werden, aber der Druck muss mehr daraus entstehen, dass das Umfeld eben was voranbringt und dass man da eben auch mittut, also ein positiver Druck.“

Wechselseitige Motivation und Inspiration werden von Natur- und IngenieurwissenschaftlerInnen also als wichtige Aspekte der Forschungsarbeit hervorgehoben. Während in den Politikwissenschaften sowohl vergemeinschaftete als auch individuelle Formen der Wissensgenerierung als sinnstiftende Forschungserfahrungen beschrieben werden, finden wir in den Literaturwissenschaften und im Strafrecht hingegen häufiger Deutungen, nach denen Erkenntnisgewinn vor allem das Ergebnis von Individualforschung ist. Dies zeigt sich etwa bei einem Strafrechtsprofessor, der seine Forschung in erster Linie als einsamen Reflexions- und Schreibprozess definiert. Was er bereits in der Promotionsphase als positiv erlebt habe, entspreche auch heute noch seinen Wünschen:

„Das sah so aus, dass ich irgendwie am Schreibtisch gesessen habe, dass ich in meinem Raum auf- und abgegangen bin und nachgedacht habe und am Ende da so ein Buch war. Und das hat, das macht mir Spaß, das macht mir auch immer noch Spaß.“(JUR6)

Er beziehe seine Mitarbeitenden zwar insofern in seine Forschungsarbeit mit ein, als er ihnen ab und an Entwürfe „zu lesen gebe“ und sie nach ihrem Feedback frage. Forschung zu einem gemeinsamen Thema komme jedoch kaum vor:

„Also zum Beispiel eine Mitarbeiterin, die gerade ausgeschieden ist, hat zu etwas Tierschutzrechtlichem geschrieben, weil das so ihr Thema ist. Davon hatte ich bis dahin keine Ahnung. Dass die Anderen am selben Projekt sitzen wie ich, nein, das ist auch bei Jura weiterhin eher nicht das Übliche.“

Für den Strafrechtler besteht in dieser Hinsicht kein Veränderungsbedarf. Er grenzt sich vielmehr von einer Forschung im Rahmen von Drittmittelprojekten ab, bei der dann Mitarbeitende „die Arbeit mach[t]en“, die er ,eigentlich immer machen wollte“, als er sich „diesen Beruf ausgesucht" habe, und die er ,auch besser" könne als jüngere NachwuchswissenschaftlerInnen. Die eigenen hohen Qualitätsansprüche, denen der Nachwuchs noch nicht genügen könne, werden auch deutlich, wenn er Negativbeispiele wie das Folgende beschreibt: 
„,[V]iele lassen ja auch schreiben und gucken sich das gar nicht an. Also es gibt zum Beispiel einen Kollegen in [Stadt], unter dessen Namen gibt es Meinungsstreitigkeiten. Der vertritt beide Meinungen, weil der so seine Mitarbeiter nicht mehr überblicken kann und der eine hat ihm das geschrieben, Meinung A, und der andere hat die Meinung Nicht-A, also genau das Gegenteil geschrieben. Kann man also für beides zitieren, denselben Herren.“

Der Strafrechtler verbindet mit einer Vergemeinschaftung der Forschungsarbeit somit nicht allein eine Distanz zu den Forschungsgegenständen, sondern auch eine Erosion des nötigen Forschungsethos.

Ein Literaturwissenschaftler grenzt sich zwar nicht in dieser Weise von KollegInnen ab, die ihre Forschung gemeinsam mit Mitarbeitenden bestreiten. Er geht jedoch ebenso in starkem Maße von einer individuellen Beschäftigung mit seinen Forschungsthemen aus:

„Die Heterogenität meiner Forschungsbereiche bisher ist nicht heterogen in meinem Inneren. Das bin ich sozusagen. Also die Addition dieser Forschungsbereiche hat viel mit meinem Temperament, meinem Charakter irgendwie zu tun, mit dem, der ich bin." (LIT1)

Der Professor beschreibt sich eindrücklich als Wissenschaftler, der sich als ganze Person mit seinen Forschungsgegenständen verbindet. Eine jüngere Fachkollegin betont zwar weniger einen solchen individualisierten Zugang zu ihren Forschungsthemen. Gleichwohl markiert sie die aus ihrer Sicht deutlichen Unterschiede zwischen geistes- und naturwissenschaftlicher Forschung. Während NaturwissenschaftlerInnen gemeinsam ,,mit einem großen Staff“ (LIT2) am Lehrstuhl forschten, brauche man für die wissenschaftliche Arbeit in ihrer Disziplin eher die Distanz zum universitären Setting:

„,[B]ei uns ist es tatsächlich so, dass die Klausur, der Rückzugsort der Forschungsschreibtisch ist gewissermaßen, der nicht der Unischreibtisch sein darf, weil man sonst zu nichts kommt. Dass der dann auch möglich ist, dass diese Freiräume möglich sind. Aber das ist, glaube ich, fächerspezifisch und gehört zu unserer Wissenschaftskultur."

Die von der Literaturwissenschaftlerin beschriebenen disziplinären Unterschiede werden zwar durch unser Datenmaterial weitgehend bestätigt. Dennoch lassen sich die professoralen Deutungen nicht auf eine Dichotomie zwischen Individualund Teamforschung reduzieren. Denn auch in der Germanistik und im Strafrecht 
finden sich Beispiele für vergemeinschaftete Forschungsprozesse. Dies trifft etwa auf jenen Literaturwissenschaftler zu, dessen Interesse für die Digital Humanities schon angesprochen wurde und der seine Forschungsarbeit folgendermaßen beschreibt:

„[D]ie klassische Germanistik, da mache ich das tatsächlich alleine. [...] Das hängt nur daran, dass ich Zeit habe, in die Bibliothek zu gehen, die Bücher aufzuschlagen ((lacht)) und meinen Laptop dabei zu haben. Und so arbeiten auch die meisten. Manchmal gibt es das, dass sie zu zweit was machen.“ (LIT4)

Währenddessen arbeite er im Bereich der Digital Humanities „sehr eng“ mit KollegInnen verschiedener Disziplinen zusammen und verfasse, wie zuletzt erst, mit diesen gemeinsame „Papers“:

„Das können wir nur als Team. Das hat vier Jahre lang Arbeit gekostet, mit Labor und so weiter. Da geht die Arbeit weiter, wenn ich aufhöre. ((lacht))“

Die für seine Disziplin eher typische Individualforschung wertet der Literaturwissenschaftler weder grundsätzlich ab noch zieht er sich aus ihr zurück. Durch sein Engagement in den Digital Humanities erlebt er jedoch zusätzlich eine stärker vergemeinschaftete Form der Wissensproduktion.

Eine Parallelität von Individual- und Teamforschung beschreibt ebenso ein Strafrechtler, der neben seiner Arbeit an Einzelpublikationen auch Drittmittelprojekte durchführt, in denen NachwuchswissenschaftlerInnen den Großteil der empirischen Arbeit leisteten. Diese gingen ,ins Land und führ[t]en die Interviews“, während er selbst vor allem für die inhaltlichen „Weichenstellungen“ (JUR10) zuständig sei. ,Gute“ Forschung bedeutet aus Sicht der beiden Professoren insofern nicht allein die individuelle Arbeit am heimischen „Forschungsschreibtisch“ (LIT2). In den Politikwissenschaften wird die gemeinsame Forschung mit Mitarbeitenden häufiger als normaler Aspekt der wissenschaftlichen Tätigkeit gedeutet. So äußert ein älterer Professor etwa, es früher ,toll“" gefunden zu haben, mit „,vier, fünf Mitarbeiter[n] in einem Projekt“" (POL6) zu arbeiten. Dass er derzeit lieber allein oder in kleineren Teams forsche, liege vor allem an seinen nun weniger groß angelegten Forschungsfragen.

Im Gegenzug zeichnen sich durchaus auch in Interviews mit Natur- und IngenieurwissenschaftlerInnen Ambivalenzen ab, wenn es um die Forschung in Arbeits- oder Projektgruppen geht. Ein wichtiger Grund dafür ist, dass sich mit der mehr koordinierenden und leitenden Rolle unweigerlich eine Distanz zu konkreten Forschungsprozessen einstellt, die das eigene Interesse an der Wissen- 
schaft zumeist erst geweckt haben. Eine ältere Zellbiologin gehört zu jenen ProfessorInnen, für die die aktive Mitarbeit an Experimenten ein besonderes Highlight geblieben ist. Sie versucht daher, die Nähe zum Labor - soweit möglich - in ihren beruflichen Alltag zu integrieren:

„Ich bin sogar, was also völlig verrückt ist, und einige lächeln darüber, ich bin sogar seit drei Jahren, aber auch vorher schon immer mal wieder, ins Labor gegangen. Ich gehe also mit einem Doktoranden hier ins Isotopenlabor. Da mache ich Dinge, die die anderen nicht so gerne machen ((lacht)), und das macht total Spaß, wenn man mal wieder selber ein Experiment machen kann.“(BIO3)

Deutlich werden in ihrer Äußerung zwei Punkte: zum einen der eindrücklich positive Bezug auf den experimentellen Charakter naturwissenschaftlicher Forschung; zum anderen aber auch die Notwendigkeit, die Arbeit im Labor als einen Freiraum gegenüber anderen Rollenerwartungen zu behaupten.

Eine jüngere Fachkollegin sieht diesbezüglich für sich kaum Möglichkeiten. Durch die Verlagerung ihrer Aufgaben zu ,,sehr viel Arbeit“ (BIO6) der Mitteleinwerbung und Nachwuchsbetreuung fehle ihr die Zeit dafür, selbst im Labor tätig zu werden, was sie als einen Verlust eigener Kompetenzen erlebt:

„Ich sehe die prozessierten Daten. Also ich bin etwas zu weit weg. Es gibt ja zwei Ebenen: Eine ist das reine Pipettieren, also das manuelle, und die Geräte bedienen können. Da bin ich schon definitiv gar nicht mehr in der Lage dazu. Das nächste ist, ob man mit den Originaldaten, die aus der Maschine kommen, ob man die auswerten kann, und das macht bei mir eine Assistentin. Das wär wahrscheinlich besser, wenn ich das auch könnte. Da müsste ich nochmal Zeit investieren, mich fortzubilden.“

Anstatt in actu dabei zu sein, geht sie ,die Versuche“ vielmehr nachträglich in regelmäßigen Meetings ,durch“. Eine gewisse Kompensation bietet ihr allerdings die Arbeit an Forschungsanträgen, die sie ,gerne“" schreibe, da man das Vorhaben jeweils „,klar gliedern und klar kommunizieren“ müsse:

„Das find ich ganz wichtig, Forschungsanträge schreiben. Also diese Forschung allein: ,Ach wir gucken mal, und mal sehen was rauskommt", also ich finde, man muss schon hypothesengetrieben arbeiten und sich überlegen, wenn das Experiment so ausgeht, welche Schlussfolgerung zulässig ist.“

Die Forschungsanträge sind in ihrer Wahrnehmung somit keineswegs eine ungeliebte Pflicht, die sie nur aus der instrumentellen Zielsetzung erfüllt, Forschungsgelder einzuwerben. Für die Zellbiologin bietet die Antragsarbeit viel- 
mehr eine wichtige Basis dafür, ihren eigenen Ansprüchen an ,gute ' Wissenschaft gerecht zu werden. Durch die Verantwortung für die Anträge kommt ihr zudem mehr als die Rolle einer Projektmanagerin zu. Indem sie die Vorannahmen, Zielsetzungen und Vorgehensweisen der Projekte definiert, nimmt sie weiterhin eine inhaltlich gestaltende Position innerhalb ihrer Arbeitsgruppe ein. ${ }^{10}$

Auch wenn es um das Thema der Zusammenarbeit mit Fachkolleginnen oder Kollegen aus anderen Disziplinen geht, werden in den Interviews Ambivalenzen sichtbar, die auf subjektive Ansprüche an die Forschung verweisen. Besonders finden wir solche Ambivalenzen erwartungsgemäß bei ProfessorInnen, die sich als IndividualforscherInnen verstehen. So zieht ein Literaturwissenschaftler klare Grenzen gegenüber gemeinsamen Projekten mit anderen ProfessorInnen:

\begin{abstract}
„[I]m geisteswissenschaftlichen Bereich gibt es nur Individualforschung. Alles andere ist sowieso Fiktion. Allerdings ist man mit solchen individuellen Ideen - und solche Ideen werden einfach nur individuell hervorgebracht - ja nicht alleine, und da braucht man in der Tat den Austausch und es kommt darauf an, dass man sich solche Strukturen des Austausches schafft.“ (LIT9)
\end{abstract}

Während er die kollegiale Vergemeinschaftung also auf einen Austausch, wie er etwa auf Tagungen stattfindet, beschränkt, bemisst er die Qualität seiner Forschung letztlich an der Zahl ,größerer Bücher“, die er allein verfasst. hat.

Ein Fachkollege von ihm äußert sich diesbezüglich differenzierter. Auch wenn er „ganz ehrlich sagen“ müsse, selbst „tendenziell gerne allein“ zu forschen, gebe es in seiner Disziplin durchaus Themen, die ,wunderbar im Kollektiv funktionier[t]en“ bzw. sich „eben wirklich nur so erforschen“ ließen. Anders als sein Fachkollege deutet er gemeinsame Projekte von GeisteswissenschaftlerInnen somit nicht per se als eine „Fiktion“. Entscheidend ist für ihn, dass Forschungskooperationen „Bottom up entwickelt“ und von einer gemeinsamen ,sachlichen Zielsetzung“ (LIT8) getrieben sind. In Interviews mit VertreterInnen anderer Disziplinen finden sich noch weit positivere Deutungen einer kollegialen Zusammenarbeit. Die schon zitierte ältere Zellbiologin betont etwa die Notwendigkeit, unterschiedliche „Experten richtig zusammenzubringen“, denn erst

\footnotetext{
${ }^{10}$ Nicolas Winterhagers (2015) vergleichende Untersuchung des Drittmittelwettbewerbs in der Biotechnologie und der Mittelalterforschung lässt diese inhaltlichen Aspekte des Antragschreibens eher unberücksichtigt und betrachtet die Einwerbung von Drittmitteln und die dazugehörigen Forschungsanträge zu einseitig hinsichtlich der strategischen Positionierung im stärker gewordenen Wettbewerb.
} 
durch die Vernetzung ,mehrere[r] Fäden“ (BIO3) sei eine wirklich umfängliche Bearbeitung ihrer Forschungsfragen möglich:

\begin{abstract}
„,[D]a kann man noch so viel rausholen, und ich habe jetzt Kooperationspartner, die glücklicherweise alle ein bisschen jünger sind, ((lacht)) in [Land], in [Land], in [Land] und auch in [Land], und mit denen wir verschiedene Aspekte um dieses Protein herum [untersuchen]. Es ist nur ein Protein, aber man kann da so viele verschiedene Aspekte [untersuchen]: Was hat es für Funktionen? Wie wandert es von da nach da? All solche Dinge. Das mache ich alles in Kooperation. [...] Dann reise ich natürlich auch sehr viel oder ich kriege viel Besuch ((lacht)). Doch, das macht schon sehr viel Spaß.“
\end{abstract}

Die gemeinsame Forschung mit FachkollegInnen aus benachbarten Teildisziplinen wie der „Biochemie“ oder der „Molekularbiologie“ bedeutet für sie einen klaren inhaltlichen Mehrwert. Anstatt miteinander zu konkurrieren, ergänzten sich die unterschiedlichen Perspektiven in produktiver Weise und bereicherten das Wissen zu dem ,einen Protein“ entscheidend.

In einigen Äußerungen zeigen sich noch stärkere interdisziplinäre Grenzüberschreitungen, die die eigene Forschungsperspektive und das eigene Fach nachhaltig verändern könnten und so als besonders erkenntnisreiche Forschungserfahrungen gedeutet werden. So beschreibt ein Professor für Nachrichtentechnik eine zwischen Ingenieur- und Naturwissenschaften bestehende Kooperation folgendermaßen:

\begin{abstract}
,[I]n dem Fall geht es um molekulare Kommunikation, ((lacht)) also Kommunikation zwischen Zellen. Man versucht das jetzt nachzubauen, ((lacht)) technisch zu realisieren. Und da lernt man ganz neue Sachen, also wie biologische Systeme funktionieren, und wie man vielleicht einige Vorgänge dort sich zunutze machen kann, auch für technische Vorgänge. Ich meine, so was ist interessanter, das reizt mich.“ (TECH13)
\end{abstract}

Den interdisziplinären Zugang deutet der Nachrichtentechniker somit im Sinne neuer Impulse für seine Forschungsperspektive und -praxis. In ähnlicher Weise argumentiert auch ein Strafrechtsprofessor, wenn er interdisziplinäre Forschung mit der Chance verbindet, eigene Prämissen kritisch zu reflektieren:

„Generell kann ich sagen, dass interdisziplinäres Arbeiten für mich signifikant bereichernder ist als das Arbeiten innerhalb der eigenen Fachdisziplin. Weil natürlich das Spektrum an potenziellen Entdeckungen viel größer ist, und der Charme eben dann darin besteht, sich auch einzulassen auf Sichtweisen anderer. Mich hat es zum Beispiel dazu gebracht, auch meine eigene Fachdisziplin zunehmend kritischer, 
also das Recht, auch in seiner Wirkweise sehr viel kritischer zu betrachten. Und zwar aus einer Sicht, die nicht nur aus der Disziplin selber herauskommt, sondern eben zunehmend auch von außen [...].“(JUR1)

Die Einbeziehung von „Einschätzungen, Selbstverständnisse[n], Haltungen“ anderer Disziplinen deutet der Strafrechtler somit nicht nur als Gewinn bei der Bearbeitung einer konkreten Forschungsfrage. Vielmehr ändere sich auch seine Perspektive auf die eigene Fachdisziplin und die gesellschaftlichen Folgen geltender Rechtsnormen. Hierdurch könne er im Gegenzug seine ,eigenen Vorschläge für rechtliche Regeln besser einschätzen“ und „adäquater" in seinem „eigenen Bereich wieder tätig“ sein.

Allerdings werden auch bei jenen ProfessorInnen, für die gemeinsame kooperative Forschungsprojekte selbstverständlich zu ihrer wissenschaftlichen Arbeit gehören, Ambivalenzen sichtbar. Ein wichtiges Thema sind dabei unterschiedliche Vorstellungen über den angemessenen Umgang mit Wettbewerbsund Zeitdruck. Die zuletzt zitierte Zellbiologin, die Kooperationen eigentlich sehr schätzt, kommt etwa auch auf negative Erfahrungen zu sprechen, die sie bei gemeinsamen Publikationen mit Projektpartnern machte. Da man für die Beantragung weiterer Forschungsmittel Veröffentlichungen nachweisen müsse, stehe man ,immer unter Hetze“ (BIO3). Unter diesem Druck hätten ihre KollegInnen schneller als sie und ihr eigenes Team Ergebnisse publizieren wollen:

\footnotetext{
„Das ist ganz furchtbar. Ich habe das bei Kooperationspartnern hier erlebt, die wollten unbedingt etwas publizieren, was ich noch gar nicht für publikationsreif hielt. Und die wollten uns da mit reinziehen natürlich. Und ich habe hier meine Mitarbeiterin, die sagt, das können wir doch noch nicht machen, das ist doch noch nicht gut. 'Weil die einen ganz anderen Anspruch haben. Den kriegen sie ja von mir. $((\text { lacht }))^{\text {“ }}$
}

Die Zellbiologin sah die Gefahr, in eine Praxis „reingezogen“ zu werden, die ihren Ansprüchen an ,gute " Forschung widersprach. Für sie ist es „furchtbar“, wenn der Qualitätsmaßstab, nur wissenschaftlich abgesicherte Ergebnisse zu veröffentlichen, verletzt wird. Sie verzichte daher ,lieber auf die Publikation“, anstatt sich dem Wettbewerb zu sehr zu unterwerfen. Ähnliche Erfahrungen aus der kooperativen Forschungsarbeit werden von einem Politikwissenschaftler geschildert. Dieser ließ sich allerdings darauf ein, ein noch „unrunde[s] Papier“ zu veröffentlichen, weil es „der Co-Autor [...] publiziert haben wollte“: 
„Da hatten wir eine Schwäche in der Theorie einfach, und es war halt das erste Papier aus diesem Projekt und da waren wir eigentlich selber noch nicht so weit, um da jetzt die sauberen Ergebnisse zu haben. Vielleicht ist es auch einfach Teil der Wissenschaft, dass sich das sozusagen immer graduell weiterentwickelt. Aber eigentlich war das zum ersten Mal, dass ich etwas publiziert habe, mit dem ich inhaltlich, substanziell inhaltlich unzufrieden war." (POL1)

Die professoralen Deutungen lassen sich insgesamt nicht auf die klaren Alternativen einer Ablehnung oder Befürwortung von Forschungskooperationen reduzieren. Solche Kooperationen können neben organisationalen Herausforderungen auch Gefährdungen von für die berufliche Identität wichtigen Qualitätsansprüchen mit sich bringen, was die erzielbaren strategischen Wettbewerbsvorteile nicht wert sind.

\section{Forschung im Spannungsfeld von Scientific Community und gesellschaftlichem Umfeld}

Bisher konnte der Eindruck entstehen, dass sich die interviewten Professorinnen und Professoren durchgängig an Grundlagenforschung und dem Bezugsrahmen der Scientific Community orientieren. Dies würde den in Kap. 2 skizzierten journalistischen Zuschreibungen entsprechen, nach denen es den meisten UniversitätsprofessorInnen vor allem um ihr Standing im wissenschaftlichen Feld und nicht etwa um einen Praxisbezug oder einen Wissenstransfer zwischen Hochschule und Gesellschaft im Sinne der vielbeschworenen „Third Mission" gehe. Allerdings zeigt sich gerade in den Interviews mit Ingenieur- und NaturwissenschaftlerInnen häufiger auch der Anspruch einer Verbindung von Forschung und praktischer Anwendung.

Besonders deutlich wird dies bei einem Nachrichtentechniker, für den ein Fokus auf reine Grundlagenforschung nur wenig sinnstiftend wäre. Er grenzt sich explizit von FachkollegInnen ab, die vor allem theoretisch arbeiteten und „halt irgendwie losgelöster von dem“ seien, ,was man vielleicht wirklich brauch[e]“:

„[I]ch sage es immer so: Wenn man dann mit den Leuten von der Industrie redet, und die sagen: ,Oh ja, der macht hier einen Haufen Sachen, aber was sollen wir damit?' Das fände ich dann irgendwie traurig, wenn jemand so etwas über mich sagen würde." (TECH1)

Diese Orientierung an den Erwartungen von Technologieunternehmen lässt sich mit der beruflichen Sozialisation erklären, da der Nachrichtentechniker vor seiner 
Berufung in der Industrie tätig war. Er genießt zwar den universitären Freiraum, mit seinen Mitarbeitenden auch ,eine theoretische Idee erstmal [...] ein wenig ausknautschen“ zu können. Gleichwohl soll Grundlagenforschung für ihn nicht zuletzt als Basis für anschließende Kooperationen mit der Industrie dienen. Ohne diese Verknüpfung bliebe es bloß eine theoretische Idee ,auf dem Papier“, die nicht ,ihren Weg“ in die praktische Anwendung finde. In ähnlicher Weise stellt der Nachrichtentechniker auch seine Ansprüche an Publikationen dar. Man müsse zwar theoretisch tiefgründige Aufsätze schreiben können, um ,auf der Wissenschaftsseite eine gewisse Reputation" zu erlangen. Noch wichtiger seien ihm aber Veröffentlichungen, ,wo derjenige, der es [lese], sofort versteh[e], was er damit anfangen" könne:

„Das ist eine andere Art zu publizieren. Ich will nicht nur meine Ergebnisse schön darstellen, sondern sie sollen weiter wandern. Das ist ein Unterschied, ob ich einfach sage: ,Wow, wie toll bin ich." Oder ob ich sage: ,Mensch, ich bin so, dass jemand anderes es verstehen kann.““

Ein Zellbiologe argumentiert hingegen etwas anders, indem er Grundlagenforschung und anwendungsorientierten Kooperationen mit Unternehmen eine ebenbürtige Bedeutung und Legitimation beimisst. ProfessorInnen müssten weiterhin „Exoten-Forschung“ betreiben können, weil es schließlich immer auch „Vordenker" brauche, die unabhängig von konkreten Nutzenerwägungen arbeiteten:

,[I]ch finde das beides in einer gewissen Weise spannend. Das eine hat einen eher praktischen, absehbaren Effekt, und das andere ist halt, wie gesagt, man weiß nicht, was dabei raus kommt. Es kann irgendwann mal einen Nutzen tragen. Aber das kann man jetzt noch nicht voraussagen. Ich würde keinem den Vorzug geben, das hat beides seine Berechtigung." (BIO2)

Gemeinsam ist beiden Professoren jedoch, bei ihren anwendungsbezogenen Projekten kein primär ökonomisches Interesse zu verfolgen. In ihren Deutungen spiegelt sich vielmehr der Anspruch eines gesellschaftlichen Nutzens ihrer Forschung wider. So macht es dem Nachrichtentechniker „Freude“, zu einer neuen Technologie beizutragen, die in die nächste, von vielen Menschen verwendete „Mobilfunkgeneration“ integriert werden könne:

„Das ist der Beitrag, den wir als Ingenieure in die Gesellschaft dann leisten können. Das heißt, jeder von uns schaltet dann wieder sein Smartphone, oder wie das Ding dann auch immer heißen mag, ein, und wenn dann so eine Technologie irgendwo ein kleines Rad ist, ist das schon mal schön." (TECH2) 
Für den Nachrichtentechniker besteht der entscheidende Gewinn somit darin, über die Kooperationen einen Wissenstransfer zu leisten, der ,in die Gesellschaft" wirke und die mobile Kommunikation für die Bürgerinnen und Bürger weiter verbessere. Auch der Zellbiologe geht bei seinen anwendungsorientierten Projekten von dem Ziel aus, zu Lösungen für gesellschaftlich relevante Probleme - wie etwa der Suche nach neuen Formen der Energiegewinnung - beizutragen. Dafür, dass er und seine KollegInnen von der „Gesellschaft bezahlt“ würden und durch ihre großen Freiheiten ,so ein Luxusleben quasi“ hätten, müsse man „,der Gesellschaft auch was zurückzugeben":

„,[U]nd das ist eben halt die Ausbildung der Kinder, und zu versuchen, eine neue Technologie zu entwickeln, [...] von der man selber keinen Nutzen hat, die aber vielleicht später dann Nutzen bringt.“ (BIO2)

Schon aufgrund der von ihm geäußerten Reziprozitätsnorm und Selbstverpflichtung gegenüber der Gesellschaft würde sich für den Zellbiologen ein ökonomisches Eigeninteresse verbieten. Er folgt einem Amtsethos, nach dem ein individueller ökonomischer Nutzen seinen NachwuchswissenschaftlerInnen vorbehalten bleibe:

„Das Projekt läuft ja weiter, und das wird von den Privatunternehmen weiter geführt, und da sind Leute von mir auch untergekommen. Das ist natürlich immer schön, wenn man hinterher sagen kann, man war da der Wegbereiter für eine Zukunft für die.“

Die beiden Fälle zeigen exemplarisch, dass Kooperationen mit der Wirtschaft keineswegs mit Deutungen und Verwertungsinteressen einhergehen müssen, die einer Kommerzialisierung von Wissenschaft entsprächen. Welche Deutungen finden sich nun aber bei Geistes- und SozialwissenschaftlerInnen, deren Forschung sich oft nicht in vergleichbar greifbarer Weise in einen gesellschaftlichen Nutzen übersetzen lässt? Nicht wenige der Äußerungen scheinen zunächst das Bild zu bestätigen, dass sich ProfessorInnen aus diesen Disziplinen primär an ihrer Scientific Community orientieren. So betont ein Strafrechtler die Bereicherung, die sich für ihn aus der Vernetzung mit Gleichgesinnten aus seinem Fachgebiet ergebe:

„Also Auftragsforschung ist irgendwie, muss man mögen, würde ich sagen. Wenn man es nicht so sehr mag, dann kommt es eben vom Thema her. Und dann trifft man in der Regel auf Kollegen, die das Thema auch gepackt hat, und das sind Aspekte, die für mich, wissenschaftlich, also im forschenden Bereich, extrem wichtig sind." (JUR2) 
Und auch eine Literaturwissenschaftlerin beschreibt vor allem ihre ,schon über Jahre“ bestehende „enge Zusammenarbeit mit einem Kollegen“(LIT13), der ähnliche Forschungsinteressen verfolge und mit dem sie gemeinsam publiziere und Fachtagungen veranstalte.

Wir finden jedoch ebenso Beispiele für eine stärkere Orientierung an einem Wissenstransfer in die Gesellschaft. So deutet es eine weitere Literaturwissenschaftlerin als Gewinn, dass sie bei einem Forschungsprojekt der letzten Jahre „sehr gut eine Verbindung zwischen Wissenschaft und Öffentlichkeit“ herstellen konnte:

„[E]inerseits sozusagen wissenschaftlich genau an einem populärkulturellen Gegenstand zu arbeiten, gleichzeitig aber ihn so aufzubereiten, dass er dann auch popularisierbar ist, die wissenschaftlichen Ergebnisse einer breiteren Öffentlichkeit vermittelbar sind, das fand ich ganz interessant. Das ist jetzt so eine Entwicklung in jüngerer Zeit. Mal sehen, ob sich das fortsetzt.“(LIT2)

In den Deutungen eines Strafrechtlers spielt der Wissenstransfer in die Gesellschaft ebenfalls eine positive Rolle. Ihm geht es dabei nicht nur um eine breitere Zugänglichkeit wissenschaftlicher Erkenntnisse, sondern gerade auch um forschungsbasierte Aufklärung. So äußert er mit Blick auf die Präsentation von Forschungsergebnissen zu den Ursachen und Mechanismen von Betrugsfällen in einem gesellschaftlich relevanten Feld:

„[I]ch fand es gut, wenn man das dann anderen Leuten erklären konnte oder präsentieren und zeigen und erklären konnte: ,Die machen das so und die machen das so, deswegen und deswegen. ' Das praktisch so ins Land zu tragen fand ich schon gut. Denn das handelte sich dann um diese hochspeziellen Fragestellungen, die für andere Leute normalerweise vollkommen neu gewesen sind und sich auch nicht ohne weiteres erschlossen haben." (JUR10)

Gemeinsam ist beiden Beispielen, dass die Vermittlung zwischen Wissenschaft und Gesellschaft und die damit verbundene Öffnung für neue AdressatInnen als positiver Teil der beruflichen Identität angeeignet wird. ${ }^{11}$

\footnotetext{
${ }^{11}$ Andere ProfessorInnen aus der Literatur- und Politikwissenschaft beschreiben etwa auch die Beteiligung an öffentlichen Ringvorlesungen oder an universitären Veranstaltungen, die sich explizit an ältere BürgerInnen oder Kinder und Jugendliche richten. Ferner sind hier noch die - wenigen, daher in unserem Sample nicht vorkommenden - ,public intellectuals“ unter den ProfessorInnen zu nennen, die vielbeachtete Artikel in Zeitungen publizieren, Kommentare in Nachrichtensendungen abgeben oder als Gäste in Talkshows auftreten.
} 
Wir finden in den Interviews jedoch auch kritische Äußerungen, die auf eine Behauptung wissenschaftlicher Prinzipien gegenüber externen - etwa wirtschaftlichen oder politischen Interessen und Handlungslogiken - abzielen. So äußern sich keineswegs nur solche ProfessorInnen, in deren Disziplinen Auftragsforschung oder Kooperationen mit Unternehmen eine ohnehin nur geringere Rolle spielen. Denn auch in der Nachrichten- und Hochfrequenztechnik wird nicht selten, wie das folgende Zitat eines Professors illustriert, eine Grenze zwischen , guter' Forschung und einer zu starken Anpassung gezogen:

„Ich kenne Fälle, die haben unglaublich viele Drittmittel, weil sie einfach verlängerte Werkbank für die Industrie machen. Die haben irgendeine Ausstattung und dann machen sie irgendwelche Messungen für die Industrie. Das hat nichts mit Wissenschaft zu tun, spielt aber natürlich viel Geld ein. Das kann nicht das Ziel einer Universität sein und es darf vor allem nicht als besonders positiv evaluiert werden." (TECH3)

Ein Fachkollege geht von einer ähnlichen Differenzierung aus, wenn er ,wirkliche Forschung"von Projekten unterscheidet, die sich zu nahe an den Interessen der Industrie bewegten:

,[I]n Deutschland ist es so: Der Großteil des Forschungsgeldes geht in irgendwelche versteckte oder nicht mal versteckte Industrieförderung, also irgendwelche Entwicklungsarbeiten für irgendwelche Industrieunternehmen. Und das bisschen Geld, wo dann wirklich Forschung stattfindet, das geht dann sowieso ans Max-PlanckInstitut. [...] Beziehungsweise dann gibt es noch die DFG. Aber bei der DFG ist es dann wirklich ein derartiges Hauen und Stechen, dass, ich weiß gar nicht, wie die Chancen liegen bei der DFG, ich glaube bei zehn Prozent oder so was." (TECH7)

Der Nachrichtentechniker kritisiert also vor allem die Struktur der Forschungsförderung. Durch diese werde eine $\mathrm{zu}$ große Nähe $\mathrm{zu}$ Industrieunternehmen forciert und steige die Zahl ,,irgendwelche[r] Entwicklungsarbeiten“ ohne wissenschaftlichen Anspruch.

Ein älterer Politikwissenschaftler bezieht sich hingegen auf die Erwartungshaltung von Akteuren aus Politik und Verwaltung, die im Falle von Auftragsforschung problematisch werden könnte. Mit Blick auf eigene Erfahrungen in der Vergangenheit wolle er zukünftig Abstand von entsprechenden Projekten nehmen:

„Es gab auch schon mal Auftragsforschung bei mir [...].Hat mir aber nicht so viel Spaß gemacht. [...] Da gab es dann auch ein Buch und so, alles schön. Aber dann war eben der Auftraggeber zufrieden mit dem was, wir gemacht haben, und das hat mich nicht so zufrieden gestellt.“ (POL6) 
Die Zufriedenheit des Auftraggebers konnte der Professor nicht teilen, da er einen zu hohen Grad der Anpassung seiner Forschung wahrnahm. Er hatte, wie er weiter ergänzt, den Eindruck, von seinem Auftraggeber letztlich für dessen Zwecke ,instrumentalisiert“ zu werden und darüber an Unabhängigkeit zu verlieren. Ein Strafrechtler kommt ebenfalls auf die Relevanz der wissenschaftlichen Unabhängigkeit zu sprechen. Die Forschung in seiner Disziplin solle zwar aktuelle Diskussionen aufgreifen und insofern einen Gesellschaftsbezug herstellen. Entscheidend bleibe aber, sich nicht daran zu orientieren, ,welcher der gesellschaftspolitischen Akteure gerade welche Akzente" setze, sondern den jeweils „eigenen Weg“ bei der Bearbeitung von Forschungsfragen „zu finden“ (JUR2). Eine Fachkollegin erweitert diesen Anspruch noch um die Forderung, sich als Wissenschaftlerin auch ,gegen den Mainstream“ (JUR3) zu stellen. Nur so könne „die Aufgabe der Universität“ gewahrt bleiben, „gesellschaftsgestaltend zu sein und nicht den Trend der Gesellschaft mitzumachen“.

\section{4 ,Gute' Lehre und, gute' Forschung: Differente Deutungen innerhalb von und zwischen Disziplinen}

In diesem Kapitel haben wir für die berufliche Identität wichtige Ansprüche an Lehre und Forschung nachgezeichnet, die wir bei unseren InterviewpartnerInnen, nicht zuletzt in Reaktion auf ihre derzeitigen Arbeitsbedingungen, vorfinden. Insbesondere in Auseinandersetzung mit Bedrohungen der so artikulierten beruflichen Identität, etwa durch „Bologna“ oder durch zu große Abhängigkeiten von Geldmitteln externer - mitunter, wissenschaftsferner" - Akteure, etwa aus der Industrie, wurden die eigenen Identitätsansprüche plastisch deutlich.

Sowohl bei den Vorstellungen zur Lehre als auch bei denen zur Forschung zeigen sich nicht unerwartete disziplinäre Unterschiede. Wichtig ist aber, dass zugleich auch ProfessorInnen derselben Teildisziplin unterschiedliche Vorstellungen ,guter' Lehre und Forschung hegen und die Fächerzugehörigkeit insofern eine wichtige, aber nicht die einzige Determinante ist. Die eigene wissenschaftliche Sozialisation, die Karrierephase und der Karriereverlauf sind ebenfalls wichtige Einflussgrößen auf die je subjektiven Ansprüche an Forschung und Lehre. ${ }^{12}$ Dies zeigte sich etwa eindrücklich im Kontext von „Bologna“: So

\footnotetext{
${ }^{12} \mathrm{Je}$ für sich genommen ist jeder dieser Faktoren in Studien schon oft betrachtet worden. Als Beispiele siehe nur Hildegard Schaeper (1997), Uta Liebeskind (2011), Andreas Franz-
} 
hielten zwar auch jüngere ProfessorInnen an Zielen wie jenem einer Integration von Forschung und Lehre fest. Anders als einige der älteren KollegInnen deuteten sie die Reformen jedoch weniger als Identitätsbedrohung, da sie sich mit ihren Ansprüchen als Lehrende nicht an einer ,besseren“, vor „Bologna“ liegenden Vergangenheit orientierten.

Wir sind ferner bei verschiedenen der angesprochenen Aspekte auf ambivalente Beurteilungen gestoßen, die dem im medialen Diskurs prominenten Schwarz-Weiß-Bild von TraditionalistInnen und ModernisiererInnen einen größeren Facettenreichtum des Geschehens entgegensetzen. So wird etwa auch von ProfessorInnen, die größeren Forschungskooperationen, wie sie nicht zuletzt durch die „Exzellenzinitiative“ propagiert wurden, offen oder positiv gegenüberstehen, darauf hingewiesen, welche Verluste eine zu weitgehende forschungspolitische Fixierung auf diesen Typus von Forschung mit sich bringen könnte oder schon gebracht hat. Umgekehrt stellen diejenigen, die den „Bologna“Reformen überwiegend kritisch gegenüberstehen, die Lehre vor „Bologna“ weder als völlig problemlos dar noch führen sie eingeräumte Probleme einzig auf die politisch verschuldete Unterfinanzierung der Universitäten zurück. Reformbedarf wird eingeräumt. Es finden sich somit keine eindeutig entweder auf früher oder auf jetzt bzw. zukünftig zugeschnittenen beruflichen Identitätsentwürfe. Früher war nicht alles gut, und vieles ist nicht oder nicht nur besser, sondern auch schlechter geworden oder auch gleich gut oder schlecht geblieben.

Wie positionieren die ProfessorInnen sich nun aber nicht nur in ihren Einstellungen, sondern in ihren auf konkrete Erfahrungen zurückgehenden beruflichen Praktiken gegenüber den Reformen von Governance, Lehre und Forschung? Wie gehen sie mit diesen Reformen und insbesondere mit neuen Formen der Leistungsbewertung um, die in diesem Kapitel noch nicht bzw. nur am Rande aufgegriffen wurden? Vor dem Hintergrund der herausgearbeiteten differenzierten Ansprüche an ,gute ' Lehre und Forschung steht zu vermuten, dass auch der tagtägliche praktische Umgang mit den durchaus als ambivalent erlebten Reformen nicht entweder in pauschaler Verweigerung oder in einer ebenso pauschalen Übernahme der Reformideen besteht, sondern sich zwischen diesen beiden Extremen ein deutlich vielschichtigeres Bild zeigt.

mann (2012) und Maximilian Fochler et al. (2016). Doch das Zusammenwirken dieser Faktoren stand bislang selten im Fokus von Untersuchungen, sodass viel Varianz, die sich aus der Kombination unterschiedlicher Ausprägungen der Faktoren ergibt, bislang übersehen wurde. 


\section{Literatur}

Bogumil, Jörg, Linda Jochheim, und Sascha Gerber. 2015. Universitäten zwischen Detailund Kontextsteuerung: Wirkungen von Zielvereinbarungen und Finanzierungsformeln im Zeitvergleich. In Hochschulgovernance in Deutschland, Hrsg. Pia Bungarten und Marei John-Ohnesorg, 55-78. Berlin: Friedrich-Ebert-Stiftung.

Bolte, Karl M., und Erhard Treutner, Hrsg. 1983. Subjektorientierte Arbeits- und Berufssoziologie. Frankfurt a. M.: Campus.

Braverman, Harry. 1977. Die Arbeit im modernen Produktionsprozeß. Frankfurt a. M.: Campus.

Dörre, Klaus. 2013. Landnahme: Unternehmen in transnationalen Wertschöpfungsketten. Aus Politik Und Zeitgeschichte 64 (1-3): 28-34.

Enders, Jürgen. 1998. Berufsbild der Hochschullehrer. In Brennpunkt Hochschule. Neuere Analysen zu Hochschule, Beruf und Gesellschaft, Hrsg. Ulrich Teichler, Hans-Dieter Daniel, und Jürgen Enders, 55-78. Frankfurt a. M.: Campus.

Fabry, Götz, und Christian Schirlo. 2016. Akademische Freiheit in professionsorientierten Studiengängen. Das Beispiel Humanmedizin. Die Hochschule: Journal Für Wissenschaft und Bildung 25 (2): 94-103.

Fochler, Maximilian, Ulrike Felt, und Ruth Müller. 2016. Unsustainable Growth, HyperCompetition, and Worth in Life Science Research: Narrowing Evaluative Repertoires in Doctoral and Postdoctoral Scientists' Work and Lives. Minerva 54: 175-200.

Franzmann, Andreas. 2012. Die Disziplin der Neugierde. Zum professionalisierten Habitus in den Erfahrungswissenschaften. Bielefeld: transcript.

Huber, Ludwig. 2014. Forschungsbasiertes, forschungsorientiertes, forschendes Lernen: Alles dasselbe? Ein Plädoyer für eine Verständigung über Begriffe und Entscheidungen im Feld forschungsnahen Lehrens und Lernens. Das Hochschulwesen 62 (1-2): 32-39.

Humboldt, Wilhelm v. 1809-10. Über die innere und äussere Organisation der höheren wissenschaftlichen Anstalten in Berlin. In Gründungstexte Johann Gottlieb Fichte, Friedrich Daniel Ernst Schleiermacher, Wilhelm von Humboldt. Festgabe zum 200-jährigen Jubiläum der Humboldt-Universität zu Berlin, Hrsg. Präsident der Humboldt-Universität zu Berlin. Berlin [2010]: Humboldt-Universität zu Berlin. https://edoc.hu-berlin.de/ bitstream/handle/18452/18543/hu_g-texte.pdf?sequence $=1 \&$ isAllowed=y.

Kern, Horst, und Michael Schumann. 1970. Industriearbeit und Arbeiterbewußtsein Frankfurt a. M.: Europäische Verlagsanstalt.

Kern, Horst, und Michael Schumann. 1984. Das Ende der Arbeitsteilung? Rationalisierung in der industriellen Produktion. München: Beck.

Liebeskind, Uta. 2011. Universitäre Lehre - Deutungsmuster von ProfessorInnen im deutsch-französischen Vergleich. Konstanz: UVK.

Loer, Thomas. 2012. Not macht erfinderisch - aber nicht in der Wissenschaft. Über die Situation der Privatdozenten und zwei Modelle von Universität. Forschung \& Lehre 19 (4): 288-289.

Lohr, Karin, Thorsten Peetz, und Romy Hilbrich. 2013. Bildungsarbeit im Umbruch. Zur Ökonomisierung von Arbeit und Organisation in Schulen, Universitäten und in der Weiterbildung. Berlin: Sigma.

Matthies, Hildegard. 2015. Die Responsivität wissenschaftlicher Karrieren. In Die Responsivität der Wissenschaft - Wissenschaftliches Handeln in Zeiten neuer Wissenschaftspolitik, Hrsg. Hildegard Matthies, Dagmar Simon, und Marc Torka, 177-208. Bielefeld: transcript. 
Mittelstraß, Jürgen. 1982. Wissenschaft als Lebensform: Reden über philosophische Orientierungen in Wissenschaft und Universität. Frankfurt a. M.: Suhrkamp.

Mittelstraß, Jürgen. 2006. Wissenschaft als Lebensform - eine Erinnerung und eine Einleitung. In Mikrokosmos Wissenschaft. Transformationen und Perspektiven, Hrsg. Brigitte Liebig, Monique Dupuis, Irene Kriesi, und Martina Peitz, 17-30. Zürich: vdf Hochschulverlag AG an der Universität Zürich.

Pasternack, Peer, Sebastian Schneider, Peggy Trautwein, und Steffen Zierold. 2017. Ausleuchtung einer Blackbox. Die organisatorischen Kontexte der Lehrqualität an Hochschulen. HoF-Arbeitsberichte (103). Wittenberg: Institut für Hochschulforschung an der Martin-Luther-Universität.

Schaeper, Hildegard. 1997. Lehrkulturen, Lehrhabitus und die Struktur der Universität. Eine empirische Untersuchung fach- und geschlechtsspezifischer Lehrkulturen. Weinheim: Deutscher Studien Verlag.

Schomburg, Harald, Choni Flöther, und Vera Wolf. 2012. Wandel von Lehre und Studium an deutschen Hochschulen - Erfahrungen und Sichtweisen der Lehrenden. Projektbericht. Kassel: Internationales Zentrum für Hochschulforschung (INCHER-Kassel), Universität Kassel.

Teichler, Ulrich. 2014. Teaching and research in Germany: The notions of university professors. In Teaching and research in contemporary higher education. Systems, activities, and rewards, Hrsg. Jung Cheol Shin, Akira Arimoto, William K. Cummings, und Ulrich Teichler, 61-87. Dordrecht: Springer.

Winterhager, Nicolas. 2015. Drittmittelwettbewerb im universitären Forschungssektor. Wiesbaden: VS.

Open Access Dieses Kapitel wird unter der Creative Commons Namensnennung 4.0 International Lizenz (http://creativecommons.org/licenses/by/4.0/deed.de) veröffentlicht, welche die Nutzung, Vervielfältigung, Bearbeitung, Verbreitung und Wiedergabe in jeglichem Medium und Format erlaubt, sofern Sie den/die ursprünglichen Autor(en) und die Quelle ordnungsgemäß nennen, einen Link zur Creative Commons Lizenz beifügen und angeben, ob Änderungen vorgenommen wurden.

Die in diesem Kapitel enthaltenen Bilder und sonstiges Drittmaterial unterliegen ebenfalls der genannten Creative Commons Lizenz, sofern sich aus der Abbildungslegende nichts anderes ergibt. Sofern das betreffende Material nicht unter der genannten Creative Commons Lizenz steht und die betreffende Handlung nicht nach gesetzlichen Vorschriften erlaubt ist, ist für die oben aufgeführten Weiterverwendungen des Materials die Einwilligung des jeweiligen Rechteinhabers einzuholen.

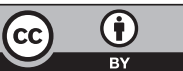

